\title{
1 Demographic Inferences and Loci Under Selection in a Recently Expanded Coral
}

\section{Population}

${ }^{1}$ Biology Department, Boston University ${ }^{2}$ Marine Biodiversity and Environmental Assessment

${ }^{2}$ Department of Marine Biology and Environmental Science, Faculty of Agriculture, University

6 of Miyazaki

${ }^{3}$ Research Center, Marine Environmental Impact Assessment Research Group, Japan Agency for

8 Marine-Earth Science and Technology

$9 \quad$ *corresponding author and lead contact: James Fifer [James.E.Fifer@gmail.com]

\section{Abstract:}

11 Coral poleward range expansions in response to warming oceans have been historically observed, however contemporary expansion rates of some coral species have become more rapid as global temperatures rise at unprecedented rates. Range expansion can lead to reduced genetic diversity and surfing of deleterious mutations in expanding populations, potentially limiting the ability for adaption and persistence in novel environments. Successful expansions that overcome these founder effects and colonize new habitat have been attributed to multiple introductions from different sources, hybridization with native populations, or rapid adaptive evolution. Here, we investigate population genomic patterns of the reef-building coral Acropora hyacinthus along a latitudinal cline that includes a well-established range expansion front in Japan using $2 \mathrm{~b}-\mathrm{RAD}$ sequencing. A total of 184 coral samples were collected across seven sites spanning from $\sim 24^{\circ} \mathrm{N}$ to near its northern range front at $\sim 33^{\circ} \mathrm{N}$. We uncover the presence of three cryptic lineages of $A$. hyacinthus, which occupy discrete areas within this region. Only one lineage is present at the expansion front and we find evidence of its historical occupation of marginal habitats. Within this lineage we also find evidence of bottleneck pressures associated with expansion events including higher clonality, increased linkage disequilibrium, and lower genetic diversity in range edge populations compared to core populations. Asymmetric migration between populations was also detected with lower migration from edge sites. Lastly, we describe genomic signatures of local adaptation potentially attributed to lower winter temperatures experienced at the more recently expanded northern populations. Together these data illuminate the genomic consequences of range expansion in a coral and highlight how adaptation to colder temperatures along the expansion front may facilitate further range expansion in this coral lineage. 


\section{Introduction:}

Populations are often locally adapted to specific environmental conditions that allow them

34 to thrive. Large-scale environment changes influence these niche conditions and can result in

35 expansion, contraction, or extinction of populations or entire species [1]. Poleward expansion in

36 response to warming oceans is a recent trend, with some species increasingly found in higher

37 latitudes (also referred to as tropicalization), specifically in regions where habitats are degraded or

38 altered [2]. These expanded populations can create socio-political tensions between states and even

39 across countries as commercially relevant species - and species that provide habitats for them -

40 modify their ranges without respect for political and sociological boundaries [3].

An expanding population can experience strong neutral and non-neutral processes. Neutral

42 founder effects can randomly change allele frequencies [4], [5] and low frequency alleles can

43 thereby 'surf' on the wave of expansion leading to high frequencies at the range front [6], [7].

44 These neutral processes can lead to reductions in genetic diversity [8]-[10], higher mutational load

45 if deleterious alleles surf [11], and false signatures of selection [5], [6], [12]. In contrast, non-

46 neutral effects associated with range expansion can produce strong selective pressures through

47 spatial sorting [13], where colonizers of new edge populations have high dispersal abilities [10],

48 [14] and/or natural selection when there is some fitness benefit to being an early colonizer [15].

49 Additionally, expansion into new environments implies that populations are exposed to novel

50 selection pressures; for poleward expansion, this entails selection pressures imposed by different

51 temperature regimes (i.e. increased seasonality) [16], [17].

52 Understanding how an organism's range responds to environmental change is particularly

53 important for species that are biological pillars for essential ecosystems, as corals are to coral reefs. 
54 Corals are some of the world's most important habitat-forming marine organisms, however their

55 recent declines are global in scale [18]. Their sensitivity to temperature [18] and rapid dispersal

56 capabilities (routinely $>10 \mathrm{~km} /$ year; reviewed in Jones et al [19]) mark corals as candidates for

57 range expansion under predicted future warming [20]. The survival of coral species under a

58 changing climate might depend on their ability to successfully shift their ranges; however, studies

59 examining coral range expansion remain scarce [21], [22]. In Japan, the coral Acropora hyacinthus

60 has been observed to expand its range [23], which has been correlated with rising temperatures in

61 the region, with temperature increases of $0.5^{\circ} \mathrm{C} /$ decade between 1982 and 2010 [24]. These

62 increases are likely mediated by the nearby Kuroshio Current's high sea surface temperature (SST)

63 warming rate (two to three times faster than the global mean SST warming rate) [25]. This ocean

64 warming has promoted macroalgal-to-coral phase shifts both directly, by increased competition

65 from the expansion of tropical corals into the contracting temperate macroalgae range, and

66 indirectly, via deforestation by the expansion of tropical herbivorous fish [26]. However,

67 historically $(\sim 5,000$ years ago) corals existed even further north than their current range limits

68 when temperatures were $2-4^{\circ} \mathrm{C}$ higher than today [27], suggesting that, like other organisms in this

69 region [28], corals may contract and expand along coastal Japan in response to glacial cycles.

71 related to their population genetic patterns. Using nuclear and mitochondrial markers, Suzuki et

72 al. [29] found that recently expanded populations showed reduced genetic diversity, which is

73 expected along an expansion front [30]. Additionally, they discovered the presence of four

74 genetically distinct $A$. hyacinthus lineages in the region. Only one of these lineages (HyaD) existed

75 within the temperate region, while all four were found in the southern Ryukyus with HyaD

76 becoming progressively rarer in southern islands. Nakabayashi et al. [31] found three genetically 
77 distinct lineages of $A$. hyacinthus using microsatellite markers and confirmed the presence of only

78 one A. hyacinthus lineage within the temperate region. This divergence between mainland Japan

79 and the Ryukyus has also been observed for a number of terrestrial (reviewed in [32]) and marine

80 species [33]-[42] between the terrestrial biogeographical barrier called the Watase line (also

81 referred to as the Tokara gap). This temperate-subtropical genetic separation has been attributed

82 to allopatric speciation during glacial maximums [39], [41]-[43], the opening and closing of

83 oceanographical barriers facilitated by shifts in the Kuroshio current during glacial maximums

84 [34], [35], [40], [43], and divergent environmental selection pressures [36], [37], [44].

Here, we examine populations of the coral A. hyacinthus near their range edge surrounding

86 mainland Japan, which includes a recently colonized site ( 40 years; [23]), and nearby populations

87 in the subtropical Ryukyus islands (Fig. 1). Using population genomics, we 1) identify $A$.

88 hyacinthus cryptic lineages and their associated distributions and demography, 2) further explore

89 the recent range expansion and core-marginal population genomic dynamics within the temperate

90 region, and 3) identify potential loci under selection on the range expansion front that might confer

91 local adaptation to differential thermal regimes experienced across the range.

92 Methods:

93 I. Sample collection, sequencing and pre-processing

\section{Coral colony sampling and $2 b-R A D$-seq library preparations}

96 were collected from seven sites in Japan between 2009 and 2016 via SCUBA (Supp. Table 1;

97 Fig. 1) under prefectural permit 21-18, 24-36, 25-44 and with agreement from local fishermen's 
98 unions. Samples were immediately preserved in $99 \%$ ethanol, stored at $-20^{\circ} \mathrm{C}$, and transferred to

99 the United States under CITES permit (13JP002699/TE, 14JP000665/TE, T-WA-12-001588, TWA-13-002421, T-WA-14-00589). DNA was isolated using a modified phenol-chloroform

101 extraction method [45], cleaned with ZYMO-DNA-clean-and-concentrator kits, and resulting

102 extracts were prepared for 2b-RAD-sequencing following Wang et al [46]. Eight replicate

103 samples were prepared to assist with clone identification. A total of 188 samples (19-40 samples

104 per site; Table 1) were successfully barcoded and sequenced across four lanes of Illumina HiSeq

105 2500, High Output v4 using single-end 50bp at Tufts University Core Facility (TUCF).

\section{Genotype calling}

108 (http://hannonlab. cshl.edu/fastx_toolkit) and only reads with Phred scores > 20 maintained.

109 Quality-filtered reads were mapped to the Acropora millepora genome [47] via bowtie2 [48].

110 Genotyping was performed using ANGSD v0.921 [49]. Standard filtering that was used across

111 all analyses included loci present in at least $80 \%$ of individuals, minimum mapping quality score

112 of 20 , minimum quality score of 25 (unless no minimum allele frequency (MAF) filter was used

113 in which case quality scores of 25 and 30 were used), strand bias p-value $>0.05$, heterozygosity

114 bias $>0.05$, removing all triallelic sites, removing reads having multiple best hits and lumped

115 paralogs filter. All analysis pipelines are open source and can be found at

116 https://github.com/jamesfifer/JapanRE.

\section{Clone identification}

118 Additional filtering was performed to detect clonemates, which included only sites with

$119 \mathrm{MAF}>0.05$, depth of coverage $>5$ reads, and SNP p-value $>0.05$. Clones were detected using 
120 hierarchical clustering of samples based on pairwise identical by state (IBS) distances calculated

121 in ANGSD. Technical replicates were used to identify appropriate height cutoff (Supp. Fig. 1).

122 Only one clonemate was retained for all downstream analyses (Supp. Table 1).

123 II. Acropora hyacinthus Lineage Analyses

\section{Lineage assignments}

Additional site filtering was performed prior to downstream analyses, which included

126 only sites with MAF > 0.05 and linked loci were filtered out using ngsLD [50] and the provided

127 prune_graph.pl script (max distance $5 \mathrm{~kb}$, minimum weight 0.2 ) to ensure population structure

128 was not driven by linkage. To detect admixture in corals from all seven sites, the program

129 ADMIXTURE (v. 1.3.0; [51]) was used to find the optimal number of clusters (K) with the least

130 cross validation error. Single Nucleotide Polymorphisms (SNPs) were hard called using

131 genotype likelihoods estimated by SAMtools with a SNP p-value < 0.05 for ADMIXTURE input

132 only. Principal Component Analysis (PCA) and admixture barplots were then visualized using

133 genotype likelihood estimates (no SNP p-values). PCAs were performed using a covariance

134 matrix based on single-read resampling calculated in ANGSD and ngsADMIX [52] and

135 admixture results were visualized using the $\mathrm{K}$ with the least cross validation error reported from

136 ADMIXTURE.

Individual lineage assignments were based on ancestral population assignments from

ngsADMIX results, where ancestral populations were only considered lineages if separation was

139 shown on the PCA first axis. If a sample had an assignment proportion $>75 \%$ to a single cluster

140 (i.e. bar color), it was assigned to that lineage. All other samples were removed from

141 downstream lineage specific analyses (Supp. Table 1). 
Analyses of genetic divergence, demographics, introgression, and phylogenetics between

\section{3 lineages}

In order to determine genetic differentiation between lineages, ANGSD was used to

145 calculate the site allele frequency (SAF) for each lineage and then realSFS calculated the site

146 frequency spectrum (SFS) for all possible pairwise comparisons. These SFSs were used as priors

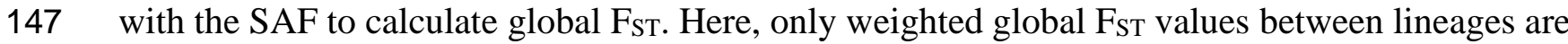

148 reported. Heterozygosity was then calculated using genotype likelihoods generated by ANGSD

149 and an expectation maximization algorithm (EM) used by realSFS in a custom script

150 (git@github.com:sbarfield/Yap_Ahyacinthus-.git/heterozygosity_beagle.r). Differences in

151 heterozygosity between lineages was calculated via Welch two sample t-test.

Linked sites were removed from downstream analyses using ngsLD and the provided

153 prune_graph.pl script (max distance 5kb, minimum weight 0.2). ANGSD was used to obtain 100

154 series of 5 block-bootstrapped SFS replicates, which were averaged to create 100 bootstrapped

155 SFS for each lineage. SFS was polarized using the A. millepora genome as an ancestral

156 reference. Multimodel inference in moments [53] was used to fit two-population models

157 (https://github.com/z0on/AFS-analysis-with-moments) and all unfolded models were run on 10

158 bootstrapped SFS and replicated six times. The best fit model was then selected based on lowest

159 AIC value. Parameters (i.e. migration, epoch times, and effective population sizes (Ne)) for the

160 best fit model were obtained by running the best fit model on 100 bootstrapped SFS and

161 replicated six times. Additionally, we ran the unsupervised analysis StairwayPlot v2 [54] to one

162 dimensional SFS as a second effort to reconstruct effective population sizes. For all demographic

163 analyses we used a mutation rate of $4 \mathrm{e}^{-9}$ per base per year and generation time of 5 years from

164 estimates for A. millepora [55], [56]. 
Phylogenetic analyses of the 2b-RAD-seq data set were conducted using RAxML [57].

166 First we explored a range of thresholds for loci coverage between samples $(50,60,70,80$ and

$16790 \%$ of individuals), MAF filter (none, $<0.001,<0.01,<0.05$ ), and minimum depth of coverage

168 (1x, 2x, 4x, 6x, 8x, 10x). RAxML, with a GTRGAMMA model and 100 rapid bootstraps, was

169 used to explore the resulting concatenated sequence matrices and choose filters that produced the

170 most reliable trees (based on node resolution and support). All trees agreed in terms of separation

171 of the three lineages and we used the standard filtering from other analyses ( $80 \%$ of individuals,

172 minimum 1x depth of coverage) plus a MAF filter $<0.05$ because this maximized bootstrap

173 support.

\section{Identifying loci under selection across lineages}

Additional filtering of loci was conducted prior to outlier analyses, which included SNP p-

176 value $\mathrm{e}^{-5}$ and MAF $<0.05$. Two outlier detection programs were used. First, PCAdapt (v.

1774.3 .3 [58]) was used to determine the optimal $\mathrm{K}$ for all pairwise comparisons using a score plot

178 displaying population structure. After filtering out two outlier individuals that were skewing

179 clustering, a $\mathrm{K}$ of 2 was selected for all pairwise comparisons between all lineage pairs. A q-

180 value of 0.05 was used as a cutoff for determining outlier loci. BayeScan (v. 2.1[59]) was then

181 used as a second approach to identify outlier loci. The same two outlier individuals were

182 removed and the FST outlier method implemented in BayeScan identified outlier loci for each

183 pairwise lineage comparison using 5,000 iterations, 20 pilot runs with length 5,000, and burn-in

184 length of 50,000. We employed the default prior odds of neutrality (10) and a q-value cut-off of

185 0.05. If the same locus was identified across both analyses, the locus was considered a true

186 outlier and the annotated genes $1 \mathrm{~kb}$ upstream or downstream of this outlier locus were reported. 
187 These genes were then compared with the module of genes previously associated with the environmental stress response (ESR) in Pacific Acropora corals [60].

\section{Analyses Within the Recently Expanded Temperate Acropora hyacinthus Lineage}

Only samples from the northern sites surrounding mainland Japan (Amakusa, Kushima,

191 Kochi, Kushimoto, and Shirahama; Fig 1), which were assigned to the temperate lineage (N=93;

192 Supp. Table 1) were used for the following analyses.

Population genetic structure, expansion direction, testing theoretical expansion consequences

To investigate basic population genetic structure within the temperate lineage, we implemented PCAs and admixture analyses, and calculated global weighted FST for all pairwise comparisons and expected and observed heterozygosities for each population. All analyses were

198 conducted as described within Section II: Acropora hyacinthus lineage analyses. The

199 PERMANOVA function Adonis (from the R package vegan [61]) was used to determine if there 200 was clustering according to core or edge. To test for differences in the number of clone pairs 201 between range edge and core populations, a Pearson's chi-squared test was used. expansion. Loci with fixed ancestral alleles were removed using SAMtools [63] and the

204 directionality index $\Psi$ was calculated using the script

205 devtools::install_github("BenjaminPeter/rangeExpansion", ref="package") and the function 206 get.all.psi. Linkage disequilibrium (LD) was estimated independently for each population using 207 ngsLD [50], and $\mathrm{r}^{2}$ was plotted for each population using fit_LDdecay.R (max 200kb). 
purifying selection, sites with synonymous and missense mutations were identified using Variant

210 Effect [64]. Global Watterson's theta was calculated for each site using the thetaStat tool in

211 ANGSD from the maximum likelihood estimate of the SFS. Watterson's theta was estimated at

212 this subset of sites for all temperate populations. We then correlated the ratio of missense to

213 synonymous site diversity with the site's distance from Kushima using Pearson's correlation

214 coefficient. Lastly, demographic analyses in moments were run as described for the coral lineage

215 analyses between all five temperate populations surrounding mainland Japan.

\section{Identifying selection pressures and loci under selection}

218 using a Mantel test with 10,000 random permutations between the FST matrix and pairwise 219 oceanographic distance. A Mantel test was also performed between the FST matrix and pairwise 220 differences in monthly minimum, maximum and mean SST. These SST data were extracted from

221 the Modis Satellite (https://oceancolor.gsfc.nasa.gov/).

223 populations within the temperate region identified using ADMIXTURE and ngsADMIX.

\section{Results:}

Analyses of population structure with ADMIXTURE and ngsAdmix using samples from

227 all sites show the presence of four $(\mathrm{K}=4)$ ancestral populations with the majority of individuals

228 assigning with high proportion (greater than 0.75) to a single population (Fig. 1). PCoA based on 
pairwise genetic distances demonstrates that these populations can be separated into three

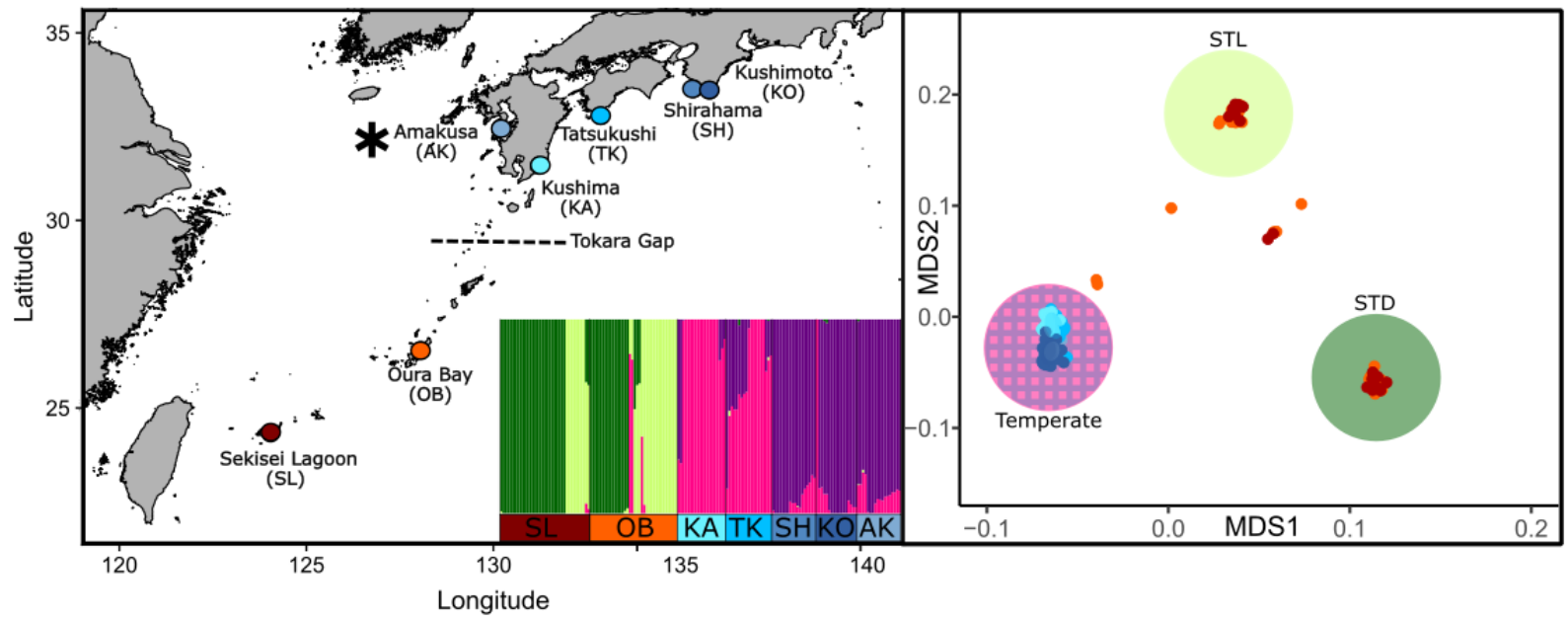

Figure 1. Left panel: Map of all coral collection sites in Japan including site names (abbreviation). Asterisk denotes recently expanded site (in the last 40 years), Amakusa. Inset: Results from Admixture analysis where each bar represents an individual coral colony and each color denotes its inferred membership to each of the 4 putative ancestral populations. Right panel: Multidimensional scaling (MDS) plot based on genetic covariance matrices of all samples, which highlights the presence of three major cryptic lineages (STL, STD, and Temperate).

\section{Pairwise $F_{S T}$ indicated high genetic differentiation $(0.1770-0.236)$ between all lineages}

(Supp. Fig. 2A) and phylogenetic analyses confirmed the presence of three phylogenetically

244 in the temperate lineage compared to the other two lineages $(\mathrm{p}<0.05)$ (Supp. Fig. 2C)

Demographic analyses for all three pairwise comparisons showed best fit models

247 analyses between all three pairwise lineage comparisons showed support for a split roughly 100 
248 kya (Fig. 2). Both subtropical lineages (STL, STD) showed asymmetrical migration with the

249 temperate lineage with higher gene flow from subtropical lineages to the temperate lineage.

250 Migration between subtropical lineages was symmetrical and it is also worth noting that STD

251 and the temperature lineage experienced an epoch with no migration (Fig. 2; Supp. Fig. 4).

252 Effective population sizes were estimated to be consistently lower in the temperate lineage when

253 compared to the two subtropical lineages (Fig. 2). Stairway plot analysis was primarily

254 consistent with the moments analysis, with the exception of the appearance of recent $\mathrm{Ne}$

255 contractions in the past few thousand years with Ne increases starting roughly 50 kyr earlier for

256 all three lineages (Fig. 3). It is noteworthy that the temperate lineage was the first lineage to

257 exhibit this contraction. 

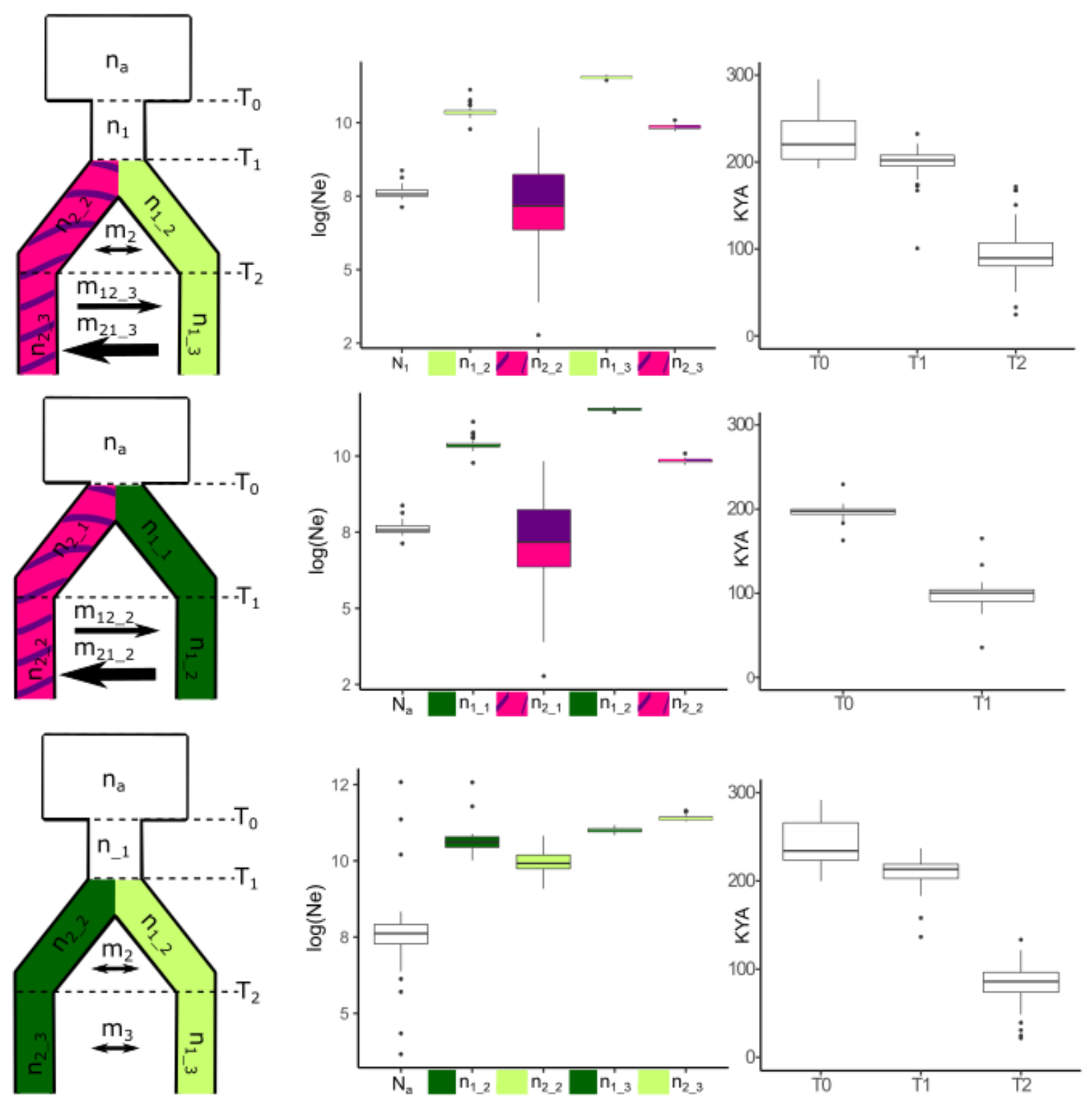

259 Figure 2. Moments parameter output for pairwise lineage analyses. $n=e f f e c t i v e$ population; $\mathrm{n} 1 \_1=$ population 1 , epoch $1 ; \mathrm{m}=$ migration. Arrow size corresponds to migration rate in case of asymmetrical migration. Boxplots display mean $\log (\mathrm{Ne})$ and mean time since introgression in kya (thousand of years from present) with the box representing the interquartile range (IQR) between the of the hinge. 

very long chain fatty acids protein 4 (EVOL4) and one unannotated gene). In the STD-STL comparison we found 56 outlier genes, 23 of which were ESR candidates (Supp. File 1).

Figure 3. Stairway Plot showing effective population sizes $\left(\mathrm{N}_{\mathrm{e}}\right)$ through time for each Acropora hyacinthus lineage. Lines show median $\mathrm{N}_{\mathrm{e}}$, ribbons represent $95 \%$ confidence intervals.

In the STL-temperate lineage comparison we identified outlier loci near 41 genes -20 of which were in the top three environmental stress response (ESR) gene modules from Dixon et al (2020). In the STD-temperate comparison we found outlier loci near 24 genes, 12 of which were ESR candidates. Five outlier genes overlapped in both temperate-subtropical lineage comparisons, three of which were ESR candidates (centrosomal protein 290kDA, elongation of

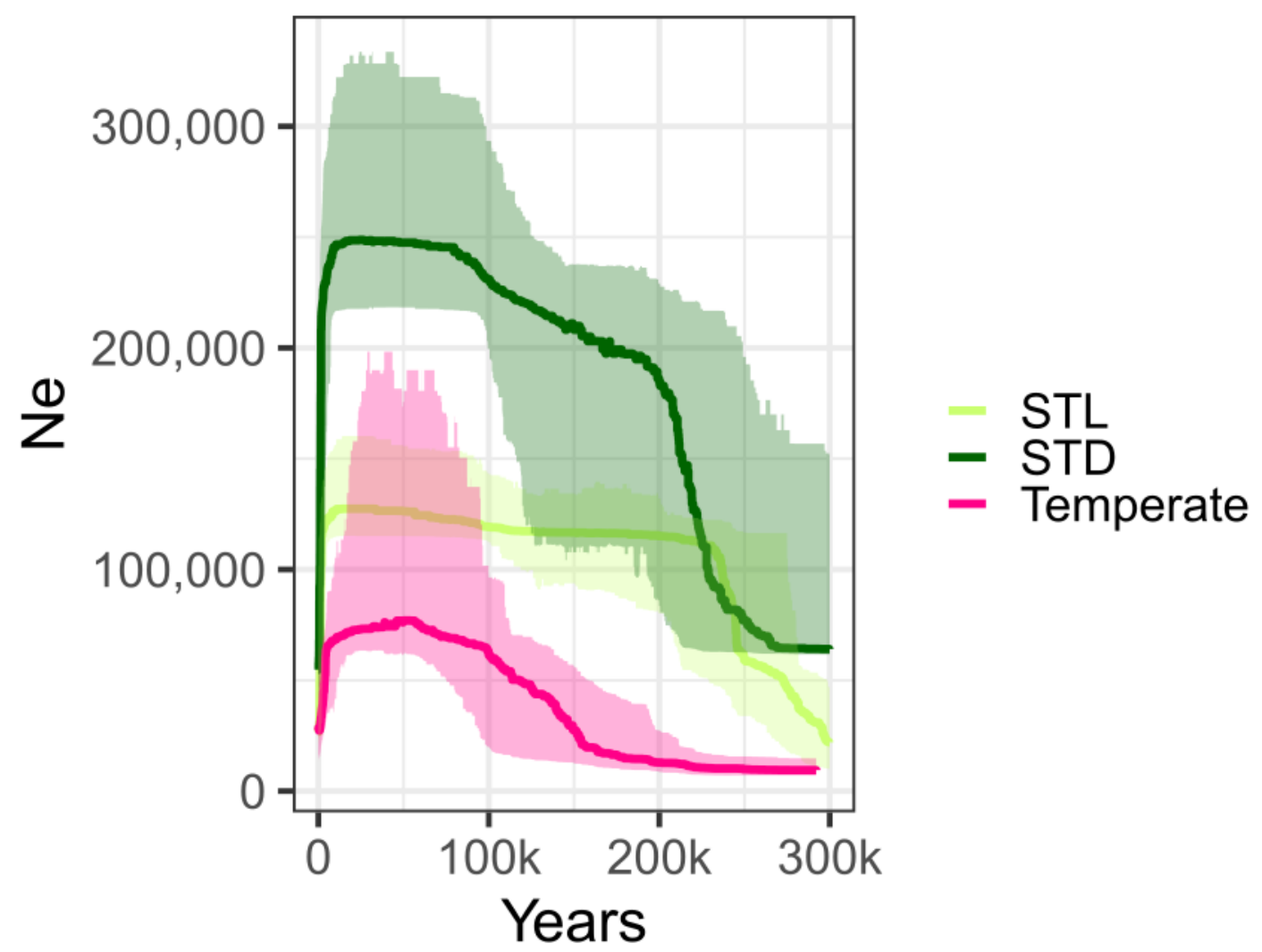

277 Genetic signature of range expansion and demographics within the temperate lineage 
Analysis of population structure with ngaAdmix and pcAngsd demonstrated separation of

279 genetic variation $(\mathrm{p}<0.001)$ between the three edge sites compared to the two core sites (Fig.

280 4A; Fig. 4B). Weighted FST estimates demonstrate low levels of differentiation among edge

281 populations (0.017-0.024) and among core populations (0.017) and higher levels of

282 differentiation between edge and core (0.036-0.045) (Fig. 4C). The directionality index $\Psi$ for

283 range expansions [62] supported the more southern sites (Kushima/Kochi) as the ancestral origin

284 for Amakusa, Shirahama and Kushimoto (Fig. 5). Expected heterozygosity was higher in core

285 populations compared to edge populations for all pairwise comparison ( $\mathrm{p}<0.05)$ (Fig. 6B).

286 Nucleotide diversity at synonymous and missense sites followed the same trend; however, edge

287 sites showed a greater decrease in nucleotide diversity at missense sites, which resulted in a

288 decreasing thetaN/thetaS ratio as populations were further from the proposed origin site Kushima

289 (Fig. 6C). This result suggests that purifying selection is stronger as distance from Kushima

290 increases (Fig. 6C). While LD was similar across all temperate populations, edge populations did

291 show a trend of higher LD (Fig. 6A). Edge populations were also home to significantly more

292 clone pairs when compared to the core populations (Pearson chi-squared $=23.969, \mathrm{df}=1, \mathrm{p}=$ 293 9.789e-07; Fig. 6B). 

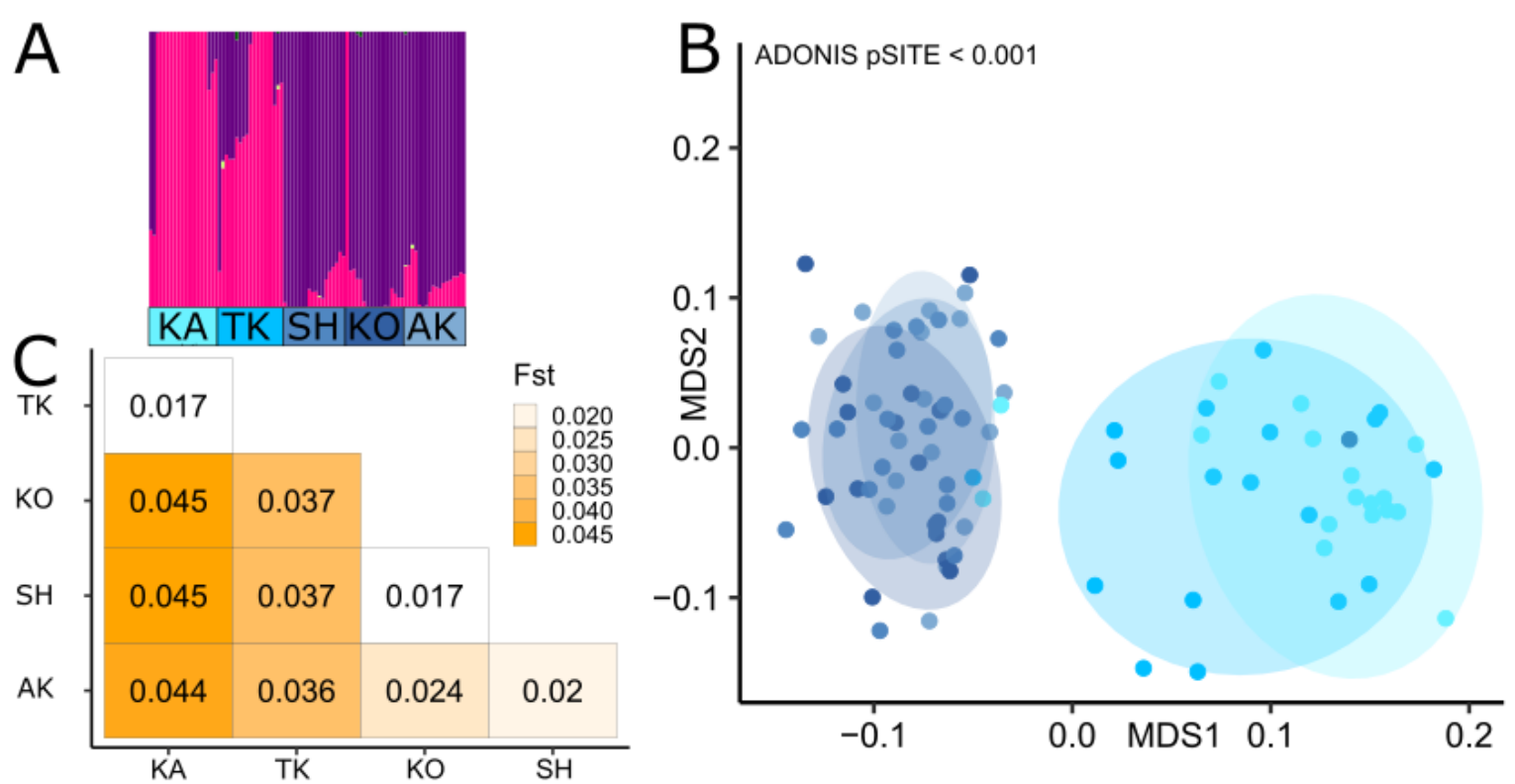

Figure 4. Population genetic structure within the temperate Acropora hyacinthus lineage. A) ngsAdmix results where each bar represents a coral individual and the color of the bar represents its inferred membership in each of the 2 potential ancestral populations within the temperate region. B) Multidimensional scaling (MDS) plot based on genetic covariance matrices demonstrating significant clustering between samples from the core sites (light blue) and the edge sites (dark blue). C) Pairwise

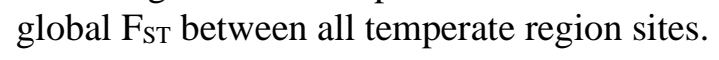

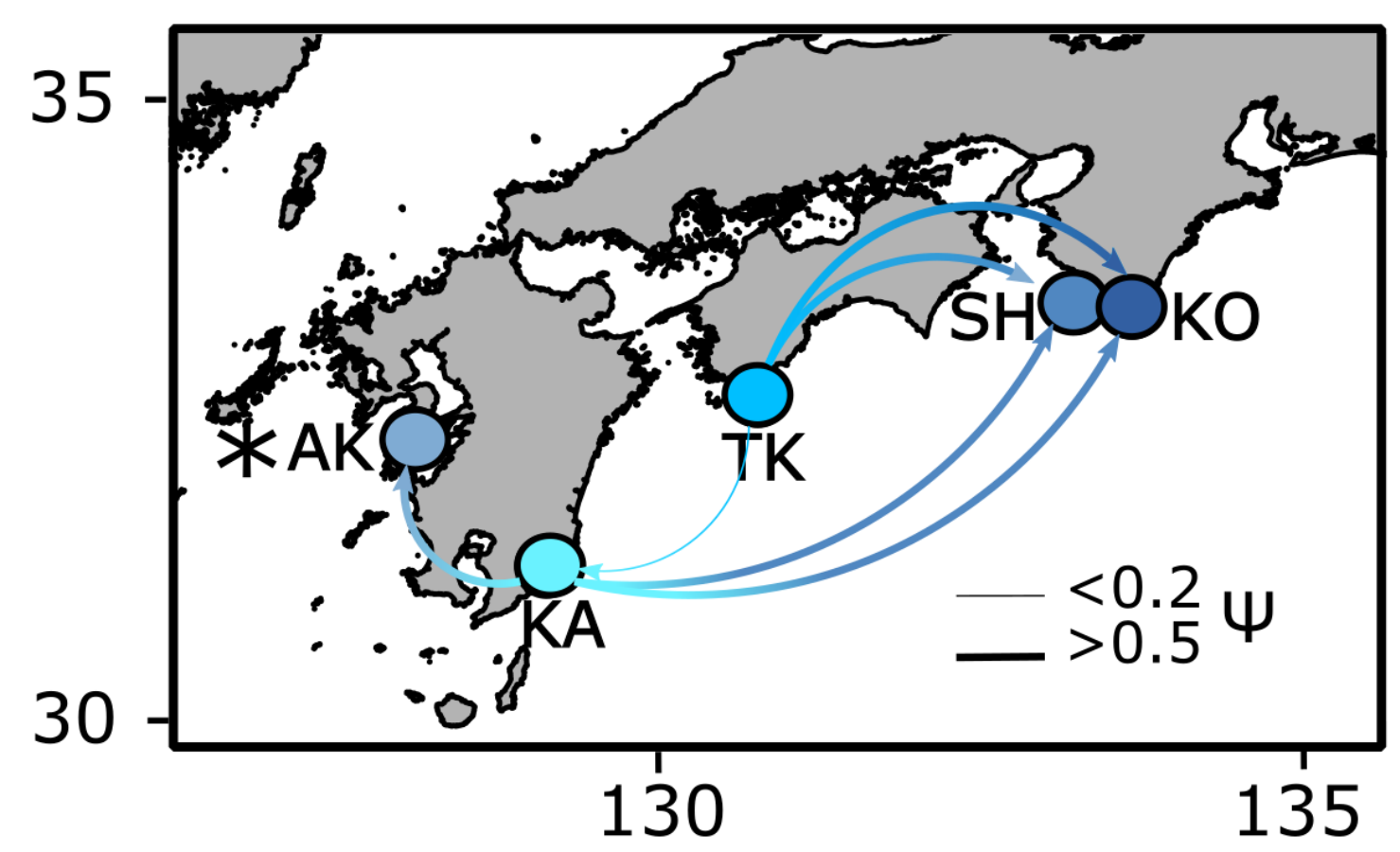

Figure 5. Migration between each temperate lineage Acropora hyacinthus population sampled in mainland of Japan, Direction of arrow denotes direction of expansion determined by positive or negative psi value. Thickness of arrow, denoting strength of signal of expansion shows absolute value of psi. Asterix indicates the most recently expanded site that was sampled here, Amakusa (AK). 


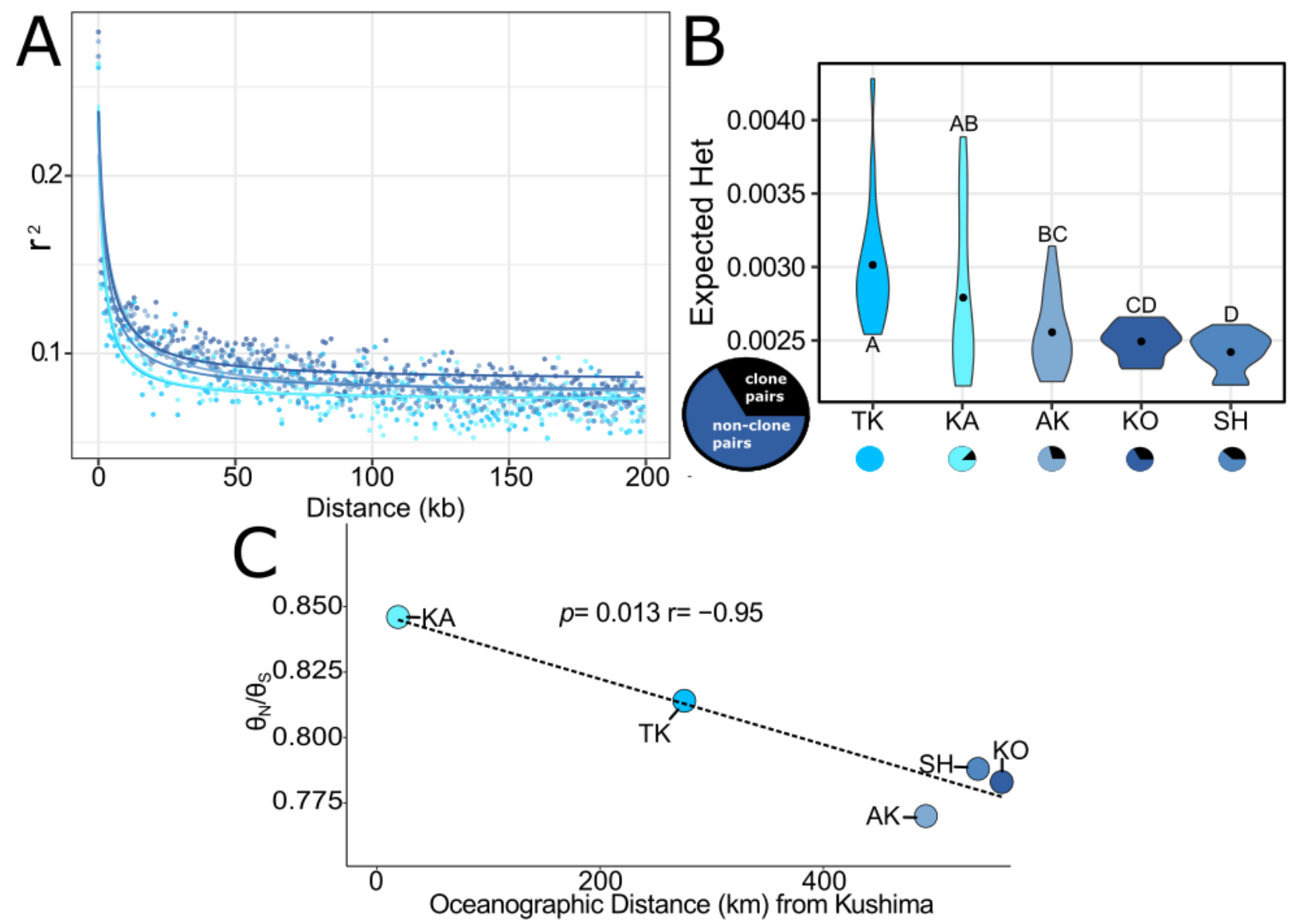

Figure 6. Genetic signatures of range expansion in the temperate Acropora hyacinthus lineage. A) Estimated linkage disequilibrium (LD) for 0-200 kb in each populations with line showing best fit decay. B) Mean expected heterozygosity $\left(\mathrm{H}_{\mathrm{e}}\right)$ estimates for each population. Letters in common denote nonsignificant differences $(\mathrm{p}>0.05)$ of $\mathrm{H}_{\mathrm{e}}$ between sites. Circles below represent the proportion of samples that were made of clone pairs (black) relative to unique genotypes (shades of blue). C) Estimated $\theta_{N} / \theta_{S}$ ratios for each population plotted against oceanographic distance from Kushima $(\mathrm{km})$ with the dotted line representing the linear model fit for purifying selection as distance increases. 

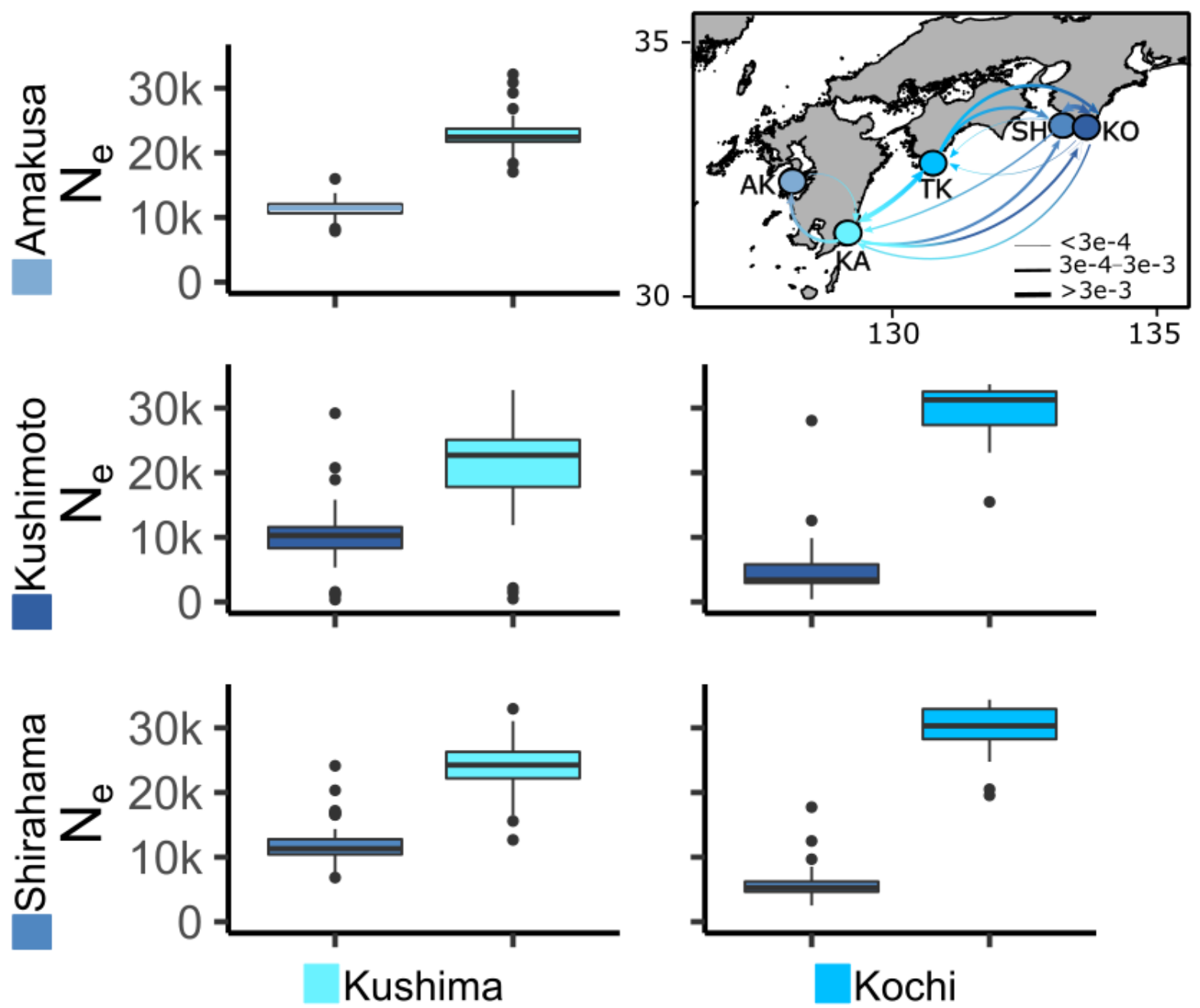

Figure 7. Pairwise moments parameter outputs for the temperate populations of Acropora hyacinthus

demonstrating mean effective population estimates $\left(\mathrm{N}_{\mathrm{e}}\right)$ and mean pairwise migration rates (the fraction of the total population that are new immigrants in each generation) between sites (shown via relative thickness of arrows, double sided arrows represent symmetrical migration).

Mantel test showed non-significant $(\mathrm{p}>0.05)$ signals of isolation by distance (IBD) and non-significant differentiation based on sea surface temperature (SST) minimums, maximums, and means, although mean and minimum SST both showed a higher correlation with F $_{\text {ST }}(\mathrm{r}=$ 


\section{Discussion:}

332

333

334

335

336

337

338

339

340

341

342

343 344 lineages STL and STD are both common in the two southern Ryukyus sites, which is consistent

345 with Nakabayashi et al [31] and Suzuki et al [29].

352

\section{Presence of Acropora hyacinthus lineages that vary in their distributions}

Consistent with previous work using different loci (microsatellites [31]; mitochondria and nuclear markers [29]), our 2b-RAD-seq data suggested the presence of three morphologically cryptic $A$. hyacinthus lineages across our sampled sites in Japan. This species is well known for its cryptic lineages with four lineages previously identified in Palau, American Samoa, and Australia, two lineages in Pohnpei and Palmyra [65], two lineages on the island of Yap in Micronesia [66], and two observed in Taiwan [29]. However, the presence of cryptic $A$. hyacinthus lineages is not always observed. For example, Davies et al. [67] found no evidence of cryptic lineages across the entire expanse of Micronesia (even in Palau) using multilocus SSR data. In addition, only one lineage has been reported in the islands of Moorea and Tahiti in French Polynesia [68], which used the same 2b-RAD-seq approach leveraged here. In Japan, latitude appears to be the main driver of the distribution of the temperate lineage, whereas

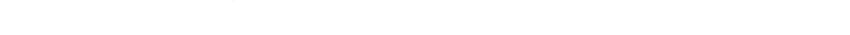

Although this study did not investigate the physiological differences between $A$.

hyacinthus lineages, coral cryptic lineages are known to exhibit differences in thermal tolerance [69] as well as differences in spawn times [70]-[72]. Interestingly, the temperate and one of the subtropical lineages have previously been shown to spawn at the same time (June) in the Ryukyus [29]. Crosses between subtropical and temperate lineages exhibited low fertilization rates but they were semi-compatible [29], suggesting that these lineages maintain some capacity for hybridization - consistent with our admixture and PCA results. Taken together, our findings 
of cryptic lineages reiterate the importance of first exploring the potential for divergent lineages

354 before investigating population connectivity, calculating speciation rates, and predicting species

355 distributions.

Demographic history of Acropora hyacinthus lineages lineages occurred during the interglacial interval around 200 kya and this divergence was facilitated by their population expansion. This result is contrary to expectation given that divergence of many other species in the region is attributed to isolation in glacial refugia of

361 which there are several in the region: Okinawa Trough, East China Sea and South China Sea

362 [73]. During interglacial periods, Acroporid corals have historically expanded populations due to

363 rising sea levels [74] and have shifted poleward as temperatures rise [75]. It is therefore possible

364 that this expansion into different regions mediated divergence between lineages; however, our

365 divergence times should be interpreted with caution as these numbers are based on an estimated

366 mutation rate. The temperate and STL lineages showed asymmetrical migration following the

367 split suggesting parapatric or sympatric speciation while the temperate lineage and STD showed

368 a period (200-100 kya) of no migration consistent with allopatric speciation. These inferences are

369 corroborated by present day distributions where the temperature lineage and only one of the

370 subtropical lineages coexist in Taiwan [29].

Given the current distributions, estimated divergence time, and $\mathrm{N}_{\mathrm{e}}$ increases by both

372 subtropical lineages following divergence, we hypothesized that the divergence of the three

373 lineages would have been facilitated by expansion into the Ryukyus. However, fossil records do

374 not support this hypothesis and instead suggest that the increase in $\mathrm{N}_{\mathrm{e}}$ was due to population 
375 growth and divergence conditioned by the introduction of a geographic barrier between the

376 Ryukyus and Taiwan/Philippines. Acroporids were present in Taiwan in the mid-Pleistocene [76]

377 and in mainland Japan in the Holocene ( 5-6 kya) [27], but no information is available

378 specifically showing the presence of A. hyacinthus in either of these regions during the

379 Pleistocene. In contrast, there are fossil records for the lower and upper Ryukyus that

380 demonstrate the presence of $A$. hyacinthus as early as mid-Pleistocene ( 400 kya) [77],

381 suggesting that the increase in $\mathrm{N}_{\mathrm{e}}$ for both subtropical lineages following the split ( 200 kya)

382 shown here does not correspond to an expansion into the Ryukyus. Given fossil records point to

383 fluctuating sea levels driving community composition shifts in the Ryukyus region [77], we

384 propose the explosion in $\mathrm{N}_{\mathrm{e}} 200$ kya for the two subtropical lineages likely captures $A$.

385 hyacinthus's population growth within these reefs, not their introduction. The absence of gene

386 flow between the temperate lineage and STL lineage, lack of increasing $\mathrm{N}_{\mathrm{e}}$ for the temperate

387 lineage following the split, and the scarcity of the temperate lineage in modern day lower and

388 middle Ryukyus still point to some degree of geographical separation between the temperate and

389 subtropical lineages 200 kya. However, our results cannot elucidate where this initial separation

390 occurred.

An increase in effective population size during the last interglacial period (100 kya) was

392 observed for all three lineages, which aligns with the pattern of major reef development in the

393 Ryukyus that responded to $100 \mathrm{kyr}$ cycles starting in the mid-Pleistocene [78]. The larger

394 increase in $\mathrm{N}_{\mathrm{e}} 100$ kya compared to 200 kya corresponds to relatively higher sea levels observed

395100 kya [78] and is consistent with data from Acropora tenuis in Japan showing increasing $\mathrm{N}_{\mathrm{e}}$

396 during the last interglacial period [79]. This result is particularly interesting as A. tenuis's range

397 overlaps with A. hyacinthus, while other Acroporids that are not found as far north as these two 
species show a decrease in effective population sizes during this time period [79]. Reduced frequencies of Acroporids in the fossil record have been observed during the last interglacial period, potentially suggesting that this group of corals experienced large die-offs during this

401 time, especially in the tropics of the Northern Hemisphere due to increasing temperatures [75].

402 Given that several Acroporids sympatrically distributed with A. hyacinthus exhibited decreasing

$403 \mathrm{~N}_{\mathrm{e}}$ during this time period [80], it is possible that these dieoffs led to niche availability, which $A$.

404 hyacinthus subsequently filled. Additionally, according to limited Pleistocene coral fossil records

405 in the Ryukyus, A. palifera (which like A. hyacinthus inhabits the shallow upper reef slope) had

406 higher abundances during the mid-Pleistocene than modern day [77]. These patterns are

407 consistent with patterns observed in Caribbean corals where increases in $\mathrm{N}_{\mathrm{e}}$ during the last

408 interglacial period have also aligned with simultaneous loss of spatially co-occurring species 409 [81].

In the case of the temperate linage, our demographic analyses suggest these populations

411 have historically inhabited marginal areas in the western North Pacific, including reefs around

412 mainland Japan. Following the increase in $\mathrm{N}_{\mathrm{e}} 100$ kya, contact was reestablished between the

413 temperate and the STD lineage, which suggests that range expansion was occurring during this

414 time period. Asymmetrical migration coupled with lower gene flow from the temperate lineage

415 to the subtropical lineage, is consistent with a scenario where the temperate lineage is

416 dominating mainland Japan habitats and due to prevailing northward currents, migrants exhibit

417 northward bias. It is therefore possible that the temperate lineage expanded its range into the

418 upper Ryukyus and around mainland Japan during this time. Lower expected heterozygosities in

419 the temperate lineage also suggest historical occupation of these marginal areas. Limited coral

420 fossil records surrounding mainland Japan can only corroborate presence of reefs as far back as 
421 the Holocene [27]. However, cold temperate kelp species - the major competitors for substrate

422 space around mainland Japan - have shifted their range under interglacial/glacial cycles [82],

423 suggesting the mechanism of modern range expansion in this region could have facilitated

424 historical range expansion of $A$. hyacinthus as well. Contractions shown in the Stairway Plot

425 analyses during the last thousand years could be due to global cooling, which started 8-10 kya in

426 the western North Pacific [83], or sudden changes in temperature during the mid-Holocene due

427 to higher variation in temperature anomalies [84]. Fossil evidence shows 5-6 kya, when SST was

$428 \sim 2^{\circ} \mathrm{C}$ higher compared to present day, reefs were prolific along the coast of Japan and found at

429 higher latitudes [27]. This range contraction aligns well with the modeled $\mathrm{N}_{\mathrm{e}}$ decline. The

430 temperate lineage's earlier contraction relative to the two other lineages is likely due to the

431 temperate lineage occupying reefs (both currently and historically) at higher latitudes than the

432 other two lineages and thus was more susceptible to cooling.

\section{Outlier Loci detected between Acropora hyacinthus lineages suggest differences in thermal}

\section{4 tolerance}

436 thermal tolerance between the temperate lineage relative to the two subtropical lineages. Five

437 overlapping outlier genes were detected in both temperate-subtropical comparisons and three of

438 these genes (two of which were annotated) have previously been implicated in the coral's

439 environmental stress response (ESR) [60]. These genes included centrosomal protein 290kDA

440 and elongation of very long chain fatty acids protein 4 (EVOL4). Centrosomal protein 290kDA

441 is involved in early and late steps in cilia formation [85] and EVOL4 is a lipid biosynthesis

442 enzyme, which has previously been shown to exhibit differential expression between coral hosts

443 infected with a homologous or heterologous algal symbiont strain [86]. One of the non-ESR 
444 outlier genes was helicase senataxin which is involved in DNA damage response generated by

445 oxidative stress [87]. Variants of helicases have also previously been found in heat resistant

446 populations of $A$. digitifera in the Ryukyus and were theorized to convey resistance against light-

447 stress associated with heat waves [88].

448 Several other outlier genes were present in only one of the two temperate-subtropical

449 lineage comparisons. Between the temperate lineage and STL lineage, ESR outlier genes

450 included Mucin-like protein and collagen alpha-5 (VI), which have both been previously

451 reported in the mineralizing matrices of mollusks [89]-[91]. Peroxidase was found to be an ESR

452 outlier gene between the temperate lineage and STD lineage and several studies have linked

453 coral peroxidase activity with anti-oxidant potential [92], [93]. Non-ESR outlier genes included

454 Fibrillin-2, which is associated with wound healing and responds to stress in Nematostella [94]

455 and other invertebrates [95] and was also identified as an outlier gene between A. tenuis lineages

456 [96]. Hemicentin-1 - another stress response wound healing gene - was found to be an ESR

457 outlier gene between the temperate lineage and STD lineage. This gene was also identified as an

458 outlier between $A$. tenuis lineages [96]. Taken together, these results suggest that the temperate

459 lineage might exhibit higher frequencies of alleles that allow for adaptation to different thermal

460 regimes, possibly facilitating this lineage's occupation of temperate habitats, which experience

461 increased seasonal temperature fluctuations.

Edge and core dynamics manifested in genomic signatures

Our genomic analyses within the temperate lineage confirm previous survey predictions

465 [23], suggesting that the direction of expansion occurred from South to North within the

466 temperate region - although we were unable to pinpoint the exact location of the origin. 
Interestingly, when exploring the assumptions of genetic consequences of range expansion we were unable to detect separation between the recently expanded Amakusa population and the range edge sites Kushimoto and Shirahama. Edge populations can share many of the same

470 characteristics as recently expanded populations including low genetic diversity [97], increased

471 LD [98], and increased selfing [99]. The high degree of similarity we observed might be because

472 the Amakusa population expanded several coral generations ago ( 40 years) and continued gene

473 flow over this time period may have dissipated the signal of a recent expansion (reviewed in

474 [100]). This potential dissipation is corroborated by a previous study of A. hyacinthus population

475 genetics in this region using microsatellites, which detected a further decrease in genetic

476 diversity in sites that expanded more recently than Amakusa [31]. Alternatively, lack of

477 separation between Amakusa and Kushimoto/Shirahama could point to a lasting signature of 478 range expansion into Kushimoto/Shirahama and additional bottlenecks driving further reduction 479 in genetic diversity in more recently expanded sites.

481 long oceanographic distance between these regions and high migration rates from core sites

482 predicted by larval dispersal models [31], could be driven by neutral forces or differences in

483 thermal regimes. For the neutral force hypothesis, edge sites could be experiencing higher drift,

484 given the evidence for asymmetrical gene flow and lower effective population sizes at these

485 sites. However, with drift's inherent randomness it seems unlikely that drift alone could produce

486 such similar genetic signatures across sites on opposite sides of mainland Japan. As the three

487 edge sites experience different thermal regimes than the two core sites, it is also possible that

488 discrete environmental selection pressures could be driving this clustering. Indeed, mantel tests

489 showed greater positive correlations between genetic distance and SST metrics compared to 
oceanographic distance, although none of these correlations were significant. This lack of

491 significance between genetic distance and SST could be caused by high migration between

492 Kushimoto and Shirahama, a non-linear relationship between genetic distance and temperature,

493 or insufficient power from our current population sample size. It is possible that high migration

494 from nearby Shirahama is driving panmixia between Kushimoto and Shirahama, however larval

495 dispersal models predict higher migration from Kushima and Kochi [31]. Alternatively, the

496 relationship between genetic distance and temperature is non-linear and instead there is some

497 winter low threshold driving the separation between the core and edge sites. However, we are

498 also limited by our small numbers of populations and effect size (i.e. Kushimoto experiences

499 SST lower than the core sites but higher than the other two edge sites). Overall, while we cannot

500 rule out neutral processes, evidence presented here is consistent with temperature-driven

501 selective forces playing a structuring role in these populations, however future physiological

502 work would need to be conducted to disentangle these hypotheses.

503

The presence of higher purifying selection detected at edge sites was counter-intuitive

504 given that theoretical [6] and empirical studies [101] predict the opposite pattern. This prediction

505 is based on the positive correlation between purifying selection and effective population size

506 (largely due to lower inbreeding in bigger populations [102]) and the observation that deleterious

507 allele surfing along an expansion front should lead to higher mutational load at edge populations

508 [103]. However, there are circumstances where edge populations can demonstrate higher

509 purifying selection. For example, bottlenecked populations can also show higher purifying

510 selection if bottlenecks are followed by $\mathrm{N}_{\mathrm{e}}$ increases [104]. Second, bottlenecks tend to purge

511 highly deleterious mutations [105], and third, if edge and core populations occupy different

512 environments, diverse selective constraints against de novo mutations could alter this signal 
513 [106]. We find the third hypothesis to be the most compelling here. Given that a large proportion

514 of the coral genome is responsive to environmental stress, and genes that underlie $\mathrm{CT}_{\min }$ or

$515 \mathrm{CT}_{\max }$ can undergo selection to remove large-effect alleles from the population by purifying

516 selection [107], this could manifest itself as stronger purifying selection genome-wide. If a

517 deleterious allele is more consequential in colder environments, this could explain higher

518 purifying selection in edge populations with exposure to suboptimal temperatures leading to

519 increased selection efficacy [108]. Future experiments elucidating thermal performance of corals

520 in this region will help elucidate the role of purifying selection in these populations.

521 To begin to explore whether selective or neutral forces are important for distinguishing

522 these genetic clusters within the temperate lineage, we performed outlier loci analyses. When

523 investigating the outlier loci between the edge population and the core population, three ESR

524 outlier genes were detected only one of which was annotated. This gene was protocadherin,

525 which is a cell-adhesion protein previously implicated in $\mathrm{pH}$ tolerance in temperate corals

526 relative to subtropical corals [109]. Two of the three non-ESR outlier genes were glutamate

527 receptors, which are well-known for their importance in circadian rhythm and day/night cycles in

528 coral [110]. These gene functions fit well into the types of environmental differences that exist

529 across latitudes and suggest that these core and edge populations within the temperate region

530 might be uniquely adapted to their respective environments.

531

532 Conclusions:

533 Our genomic analyses provide insight into the evolution and ecology of cryptic $A$.

534 hyacinthus lineages in Japan and the mechanisms that might underly the observed population

535 structure between edge and core populations at this species' range edge. We found that cryptic 
536 lineages were formed in the mid-Pleistocene and have been punctuated by large increases in $\mathrm{N}_{\mathrm{e}}$

537 that correspond with interglacial periods. Previous work has suggested that ecological

538 opportunity from changing sea levels facilitated the adaptive radiation of Acroporids [79] and we

539 show evidence to suggest that this also holds for intra-specific radiations as well. Within the

540 temperate lineage we show evidence for historical occupation of marginal sites and population

541 structure that corresponds to degrees of habitat marginalization, potentially demonstrating this

542 lineage's unique ability to continually expand north. Future work characterizing the

543 physiological differences between lineages and populations will be necessary to ground truth

544 inferences of temperature adaptation detected here. Overall, this work helps build on the growing

545 research in this region that will be critical for evaluating the resilience of Japan's reefs and

546 predicting future range expansion.

547 Data Availability:

548 Raw read data can be found on the sequence read archive (SRA BioProject accession number:

549 PRJNA735187).

550 Author Contributions:

551 SWD conceived of the project and oversaw project development. NY completed all collections

552 including permits. JEF prepared all sequencing libraries and conducted all analyses. TY provided

553 temperature data. JEF wrote the manuscript with contributions from SWD. All authors edited

554 and approved the final version.

555 Acknowledgements:

556 Akira Iguchi, Takashi Nakamura, and Mikhail Matz facilitated collections, permits and shipping

557 of samples from Japan. We acknowledge Laura Tsang, Tiffany Wong, and Brianna Regan who

558 helped isolate all DNA samples, which was overseen by Nicola Kriefall. We are grateful to 
559 Mikhail Matz, JP Rippe, Chris Schmitt, Peter Buston, Mike Sorenson, Groves Dixon, Nicole

560 Adams, Hanny Rivera, Sara Smith and Ekaterina Noskova for their assistance during analyses

561 associated with the project. Thanks to Nicola Kriefall, Hannah Aichelman, Daniel Wuitchik,

562 Colleen Bove, Lucas Fifer and Jill Grose-Fifer for comments on writing and figures. We

563 acknowledge Boston University’s SCC who facilitated all computational work.

\section{Funding Statement:}

565 Funding for this project came from startup funds to SWD from Boston University and was

566 supported by the Environment Research and Technology Development Fund (4RF 1501 and 4-

567 1304) of the Ministry of the Environment, Japan, Grant-in-aid for young scientists (A)

568 (17H04996). JF also received a Boston University Warren Mcleod award associated with this

569 research.

570

571 Declaration of interests:

572 The authors declare no competing interests.

573 


\section{References}

575 [1] J. M. J. Travis, "Climate change and habitat destruction: A deadly anthropogenic

576 cocktail,” Proc. R. Soc. B Biol. Sci., vol. 270, no. 1514, pp. 467-473, 2003.

577 [2] R. Hickling, D. B. Roy, J. K. Hill, R. Fox, and C. D. Thomas, "The distributions of a wide 578 range of taxonomic groups are expanding polewards," Glob. Chang. Biol., vol. 12, no. 3, $579 \quad$ pp. $450-455,2006$.

580 [3] M. L. Pinsky, G. Reygondeau, R. Caddell, J. Palacios-Abrantes, J. Spijkers, and W. W. L. Cheung, "Preparing ocean governance for species on the move," Science (80-. )., vol. 360, no. 6394, pp. 1189-1191, 2018.

[4] O. Hallatschek, P. Hersen, S. Ramanathan, and D. R. Nelson, "Genetic drift at expanding

[5] L. Excoffier and N. Ray, "Surfing during population expansions promotes genetic revolutions and structuration," Trends Ecol. Evol., vol. 23, no. 7, pp. 347-351, 2008.

[6] S. Klopfstein, M. Currat, and L. Excoffier, "The fate of mutations surfing on the wave of a range expansion,” Mol. Biol. Evol., vol. 23, no. 3, pp. 482-490, 2006. Biol., vol. 73, no. 1, pp. 158-170, 2008.

P. C. Watts, S. Keat, and D. J. Thompson, "Patterns of spatial genetic structure and 
[9] C. J. Garroway, J. Bowman, G. L. Holloway, J. R. Malcolm, and P. J. Wilson, “The genetic signature of rapid range expansion by flying squirrels in response to contemporary climate warming," Glob. Chang. Biol., vol. 17, no. 5, pp. 1760-1769, 2011.

[10] J. Swaegers, J. Mergeay, A. Van Geystelen, L. Therry, M. H. D. Larmuseau, and R. Stoks, "Neutral and adaptive genomic signatures of rapid poleward range expansion," Mol. Ecol., vol. 24, no. 24, pp. 6163-6176, 2015.

[11] S. Peischl, I. Dupanloup, M. Kirkpatrick, and L. Excoffier, "On the accumulation of deleterious mutations during range expansions," Mol. Ecol., vol. 22, no. 24, pp. 59725982, 2013.

[12] C. A. Edmonds, A. S. Lillie, and L. L. Cavalli-Sforza, "Mutations arising in the wave front of an expanding population," Proc. Natl. Acad. Sci. U. S. A., vol. 101, no. 4, pp. 975-979, 2004.

[13] R. Shine, G. P. Brown, and B. L. Phillips, “An evolutionary process that assembles phenotypes through space rather than through time," Proc. Natl. Acad. Sci. U. S. A., vol. 108, no. 14, pp. 5708-5711, 2011.

[14] J. K. Hill, H. M. Griffiths, and C. D. Thomas, "Climate change and evolutionary adaptations at species' range margins,” Annu. Rev. Entomol., vol. 56, pp. 143-159, 2011.

613 [15] J. M. J. Travis and C. Dytham, "Dispersal evolution during invasions," Evol. Ecol. Res., $614 \quad$ vol. 4, no. 8, pp. 1119-1129, 2002. 
thermal niche breadth results from thermal release during a climate-mediated range expansion,” J. Biogeogr., vol. 42, no. 10, pp. 1953-1963, 2015.

[17] R. Y. Dudaniec, C. J. Yong, L. T. Lancaster, E. I. Svensson, and B. Hansson, "Signatures of local adaptation along environmental gradients in a range-expanding damselfly (Ischnura elegans)," Mol. Ecol., vol. 27, no. 11, pp. 2576-2593, 2018.

[18] T. P. Hughes et al., "Global warming and recurrent mass bleaching of corals," Nature, 2017.

[19] G. P. Jones et al., "Larval retention and connectivity among populations of corals and reef fishes: History, advances and challenges," Coral Reefs, vol. 28, no. 2, pp. 307-325, 2009.

[20] IPCC, Intergovernmental Panel on Climate Change, Climate Change 2014: Synthesis Report. Geneva, Switzerland, 2014.

[21] K. P. Leydet, C. G. B. Grupstra, R. Coma, M. Ribes, and M. E. Hellberg, "Host-targeted RAD-Seq reveals genetic changes in the coral Oculina patagonica associated with range

[22] A. NAKABAYASHI, T. MATSUMOTO, Y. F. KITANO, S. NAGAI, and N. YASUDA, range expansion due to climate change?," Galaxea, J. Coral Reef Stud., vol. 19, no. 1, pp. 
637 [24] F. P. Lima and D. S. Wethey, "Three decades of high-resolution coastal sea surface temperatures reveal more than warming," Nat. Commun., vol. 3, 2012.

639

640

641

642

643

644

645

646

647

648

649

650

651

652

653

654

655

656

657

[25] L. Wu et al., "Enhanced warming over the global subtropical western boundary currents," Nat. Clim. Chang., vol. 2, no. 3, pp. 161-166, 2012.

[26] N. H. Kumagai, J. G. Molinos, H. Yamano, S. Takao, M. Fujii, and Y. Yamanaka, “Ocean currents and herbivory drive macroalgae-to-coral community shift under climate warming," Proc. Natl. Acad. Sci. U. S. A., vol. 115, no. 36, pp. 8990-8995, 2018.

[27] J. E. N. Veron, "Environmental control of Holocene changes to the world's most northern hermatypic coral outcrop,” Pacific Sci., vol. 46, no. 4, pp. 405-425, 1992.

[28] E. K. Han et al., “A disjunctive marginal edge of evergreen broad-leaved oak (Quercus gilva) in East Asia: The high genetic distinctiveness and unusual diversity of Jeju Island populations and insight into a massive, independent postglacial colonization," Genes (Basel)., vol. 11, no. 10, pp. 1-21, 2020.

[29] G. Suzuki et al., "Genetic evidence of peripheral isolation and low diversity in marginal populations of the Acropora hyacinthus complex," Coral Reefs, vol. 35, no. 4, pp. 1419$1432,2016$.

[30] L. Excoffier, M. Foll, and R. J. Petit, "Genetic Consequences of Range Expansions," Annu. Rev. Ecol. Evol. Syst., vol. 40, no. 1, pp. 481-501, 2009.

[31] A. Nakabayashi et al., "The potential role of temperate Japanese regions as refugia for the coral Acropora hyacinthus in the face of climate change," Sci. Rep.

[32] S. Komaki and T. Igawa, "The widespread misconception about the Japanese major 
biogeographic boundary, the 3 Watase line (Tokara gap), revealed by bibliographic and

beta diversity analyses,”信阳师范学院, vol. 1, no. 1, pp. 287-295, 2017.

660

661

662

663

664

665

666

667

668

669

670

671

672

673

674

675

676

677

678
[33] T. Mukai, T. Suzuki, and M. Nishida, "Genetic and geographical differentiation of Pandaka gobies in Japan," Ichthyol. Res., vol. 51, no. 3, pp. 222-227, 2004.

[34] S. Y. V. Liu, T. Kokita, and C. F. Dai, "Population genetic structure of the neon damselfish (Pomacentrus coelestis) in the northwestern Pacific Ocean," Mar. Biol., vol. 154, no. 4, pp. 745-753, 2008.

[35] S. Kojima, S. Kamimura, A. Iijima, T. Kimura, T. Kurozumi, and T. Furota, "Molecular phylogeny and population structure of tideland snails in the genus Cerithidea around Japan,” Mar. Biol., vol. 149, no. 3, pp. 525-535, 2006.

[36] N. Yasuda, S. Nagai, M. Hamaguchi, K. Okaji, K. GÉrard, and K. Nadaoka, “Gene flow of Acanthaster planci (L.) in relation to ocean currents revealed by microsatellite analysis,” Mol. Ecol., vol. 18, no. 8, pp. 1574-1590, 2009.

[37] D. Albinsky, D. Wham, N. Shinzato, and J. D. Reimer, "Population Connectivity in the Common Reef Zoantharian Zoanthus sansibaricus (Anthozoa: Hexacorallia) in Southern Japan,” Zoolog. Sci., vol. 35, no. 4, pp. 321-329, 2018.

[38] L. M. Tsang, B. K. K. Chan, K. Y. Ma, and K. H. Chu, "Genetic differentiation, hybridization and adaptive divergence in two subspecies of the acorn barnacle Tetraclita japonica in the northwestern Pacific," Mol. Ecol., vol. 17, no. 18, pp. 4151-4163, 2008.

[39] J. Xu, T. Y. Chan, L. M. Tsang, and K. H. Chu, "Phylogeography of the mitten crab Eriocheir sensu stricto in East Asia: Pleistocene isolation, population expansion and 
secondary contact," Mol. Phylogenet. Evol., vol. 52, no. 1, pp. 45-56, 2009.

680

681

682

683

684

685

686

687

688

689

690

691

692

693

694

695

696

697

698

699

[40] K. Kuriiwa, S. N. Chiba, H. Motomura, and K. Matsuura, "Phylogeography of Blacktip Grouper, Epinephelus fasciatus (Perciformes: Serranidae), and influence of the Kuroshio Current on cryptic lineages and genetic population structure," Ichthyol. Res., vol. 61, no. 4, pp. 361-374, 2014.

[41] L. He, T. Mukai, K. Hou Chu, Q. Ma, and J. Zhang, "Biogeographical role of the Kuroshio Current in the amphibious mudskipper Periophthalmus modestus indicated by mitochondrial DNA data," Sci. Rep., vol. 5, no. September, pp. 1-12, 2015.

[42] Y. W. Chang et al., "Genetic differentiation of the soft shore barnacle Fistulobalanus albicostatus (Cirripedia: Thoracica: Balanomorpha) in the West Pacific,” Mar. Ecol., vol. 38, no. 2, pp. 1-10, 2017.

[43] D. Yamazaki et al., "Genetic diversification of intertidal gastropoda in an archipelago: the effects of islands, oceanic currents, and ecology," Mar. Biol., vol. 164, no. 9, 2017.

[44] T. Takeuchi, T. Masaoka, H. Aoki, R. Koyanagi, M. Fujie, and N. Satoh, "Divergent northern and southern populations and demographic history of the pearl oyster in the western Pacific revealed with genomic SNPs,” Evol. Appl., vol. 13, no. 4, pp. 837-853, 2020.

[45] S. W. Davies et al., "Novel polymorphic microsatellite markers for population genetics of the endangered Caribbean star coral, Montastraea faveolata," Mar. Biodivers., vol. 43, no. 2, pp. 167-172, 2013.

[46] S. Wang, E. Meyer, J. K. Mckay, and M. V. Matz, “2b-RAD: A simple and flexible 
method for genome-wide genotyping," Nat. Methods, vol. 9, no. 8, pp. 808-810, 2012.

[47] Z. L. Fuller et al., "Population genetics of the coral Acropora millepora : Towards a genomic predictor of bleaching," 2019.

[48] B. Langmead and S. Salzberg L., "Fast gapped-read alignment with Bowtie 2," Nat. Methods, vol. 9, no. 4, pp. 357-359, 2013.

[49] T. S. Korneliussen, A. Albrechtsen, and R. Nielsen, "ANGSD: Analysis of Next Generation Sequencing Data,” BMC Bioinformatics, vol. 15, no. 1, pp. 1-13, 2014.

[50] E. . Fox, A. . Wright, M. Fumagalli, and G. Vieira, "ngsLD: evaluating linkage disequilibrium using genotype likelihoods," Bioinformatics, pp. 1-22, 2019. proportions from next generation sequencing data," Genetics, vol. 195, no. 3, pp. 693$702,2013$. History of Multiple Populations : Beyond the Diffusion Approximation," Genetics, vol. 206, no. July, pp. 1549-1567, 2017.

[54] X. Liu and Y. X. Fu, "Stairway Plot 2: demographic history inference with folded SNP frequency spectra," Genome Biol., vol. 21, no. 1, pp. 1-9, 2020. 
721 [56] Z. T. Richards, D. J. Miller, and C. C. Wallace, "Molecular phylogenetics of

722

723

724

725

726

727

728

729

730

731

732

733

734

735

736

737

738

739

740

741 geographically restricted Acropora species: Implications for threatened species conservation,” Mol. Phylogenet. Evol., vol. 69, no. 3, pp. 837-851, 2013.

[57] A. Stamatakis, "RAxML version 8: A tool for phylogenetic analysis and post-analysis of large phylogenies," Bioinformatics, vol. 30, no. 9, pp. 1312-1313, 2014.

[58] K. Luu, E. Bazin, and M. G. B. Blum, "pcadapt: an R package to perform genome scans for selection based on principal component analysis," Mol. Ecol. Resour., vol. 17, no. 1, pp. 67-77, 2017.

[59] M. Foll, “BayeScan v2.1,”Ecology, vol. 20, no. 11, pp. 1450-1462, 2012.

[60] G. Dixon, E. Abbott, and M. Matz, "Meta-analysis of the coral environmental stress response: Acropora corals show opposing responses depending on stress intensity," Mol. Ecol., vol. 29, no. 15, pp. 2855-2870, 2020.

[61] A. J. Oksanen et al., "Package 'vegan, ," 2020.

[62] B. M. Peter and M. Slatkin, "Detecting range expansions from genetic data," Evolution (N. Y)., vol. 67, no. 11, pp. 3274-3289, 2013.

[63] H. Li et al., "The Sequence Alignment/Map format and SAMtools," Bioinformatics, vol. 25, no. 16, pp. 2078-2079, 2009.

[64] W. McLaren et al., "The Ensembl Variant Effect Predictor," Genome Biol., vol. 17, no. 1, pp. 1-14, 2016.

[65] J. T. Ladner and S. R. Palumbi, "Extensive sympatry, cryptic diversity and introgression throughout the geographic distribution of two coral species complexes," Mol. Ecol., vol. 
21, no. 9, pp. 2224-2238, 2012.

743 [66] S. Barfield, S. W. Davies, and M. V Matz, "Co-recruitment of relatives leads to emergence of an inbred genetically isolated group within a panmictic population of a broadcast-spawning reef-building coral Sarah Barfield, Sarah W. Davies, Mikhail V. Matz University of Texas at Austin."

747 [67] S. W. Davies, E. A. Treml, C. D. Kenkel, and M. V. Matz, "Exploring the role of Micronesian islands in the maintenance of coral genetic diversity in the Pacific Ocean," Mol. Ecol., vol. 24, no. 1, pp. 70-82, 2015.

[68] N. G. Kriefall, M. R. Kanke, G. V. Aglyamova, and S. W. Davies, "Reef environments shape microbial partners in a highly connected coral population," bioRxiv, 2020.

[69] M. Gómez-Corrales and C. Prada, "Cryptic lineages respond differently to coral bleaching," Mol. Ecol., vol. 29, no. 22, pp. 4265-4273, 2020.

[70] N. L. Rosser, “Asynchronous spawning in sympatric populations of a hard coral reveals cryptic species and ancient genetic lineages," Mol. Ecol., vol. 24, no. 19, pp. 5006-5019, 2015.

[71] M. Furukawa, S. Ohki, S. Kitanobo, H. Fukami, and M. Morita, "Differences in spawning time drive cryptic speciation in the coral Acropora divaricata," Mar. Biol., vol. 167, no. 11 , pp. $1-10,2020$. the speciation of the broadcast spawning corals Acropora digitifera and the cryptic species Acropora sp. 1 with similar gamete recognition systems," Coral Reefs, vol. 34, no. 4, pp. 
1189-1198, 2015.

764 [73] P. Wang, "Response of Western Pacific marginal seas to glacial cycles:

Paleoceanographic and sedimentological features," Mar. Geol., vol. 156, no. 1-4, pp. 5-

766

39, 1999.

[74] W. Renema et al., "Are coral reefs victims of their own past success ?," Sci. Adv., no.

768 April, pp. 1-7, 2016.

[75] W. Kiessling, C. Simpson, B. Beck, H. Mewis, and J. M. Pandolfi, "Equatorial decline of reef corals during the last Pleistocene interglacial," Proc. Natl. Acad. Sci. U. S. A., vol. 109, no. 52, pp. 21378-21383, 2012.

S. Y. Gong, S. W. Wang, and T. Y. Lee, "Pleistocene coral reefs associated with claystones, southwestern Taiwan," Coral Reefs, vol. 17, no. 3, pp. 215-222, 1998.

M. Humblet, Y. Iryu, and T. Nakamori, "Variations in Pleistocene coral assemblages in space and time in southern and northern Central Ryukyu Islands, Japan," Mar. Geol., vol. 259, no. 1-4, pp. 1-20, 2009.

777 [78] N. Sagawa, T. Nakamori, and Y. Iryu, "Pleistocene reef development in the southwest Ryukyu Islands, Japan,” Palaeogeogr. Palaeoclimatol. Palaeoecol., vol. 175, no. 1-4, pp.

[79] Y. Mao, E. P. Economo, and N. Satoh, "The Roles of Introgression and Climate Change in the Rise to Dominance of Acropora Corals," Curr. Biol., vol. 28, no. 21, pp. 33733382.e5, 2018. 
Ryukyu Islands, Japan,”Sci. reports Tohoku Univ. Second Ser. Geol., vol. 56, 1986.

785
[81] C. Prada et al., "Empty Niches after Extinctions Increase Population Sizes of Modern Corals," Curr. Biol., vol. 26, no. 23, pp. 3190-3194, 2016.

[82] J. Assis, A. V. Lucas, I. Bárbara, and E. Á. Serrão, "Future climate change is predicted to shift long-term persistence zones in the cold-temperate kelp Laminaria hyperborea," Mar. Environ. Res., vol. 113, pp. 174-182, 2016.

[83] V. D. Meyer, L. Max, J. Hefter, R. Tiedemann, and G. Mollenhauer, "Glacial-to-Holocene evolution of sea surface temperature and surface circulation in the subarctic northwest Pacific and the Western Bering Sea,” Paleoceanography, vol. 31, no. 7, pp. 916-927, 2016.

[84] R. Asami et al., "High-Resolution Evidence for Middle Holocene East Asian Winter and Summer Monsoon Variations: Snapshots of Fossil Coral Records,” Geophys. Res. Lett., vol. 47, no. 16, pp. 1-11, 2020.

[85] T. Avidor-Reiss and M. R. Leroux, "Shared and distinct mechanisms of compartmentalized and cytosolic ciliogenesis," Curr. Biol., vol. 25, no. 23, pp. R1143R1150, 2015.

[86] J. L. Matthews et al., "Optimal nutrient exchange and immune responses operate in partner specificity in the cnidarian-dinoflagellate symbiosis," Proc. Natl. Acad. Sci. U. S. A., vol. 114, no. 50, pp. 13194-13199, 2017.

[87] A. Suraweera et al., "Senataxin, defective in ataxia oculomotor apraxia type 2, is involved in the defense against oxidative DNA damage," J. Cell Biol., vol. 177, no. 6, pp. 969-979, 
[88] O. Selmoni, E. Rochat, G. Lecellier, V. Berteaux-Lecellier, and S. Joost, "Seascape genomics as a new tool to empower coral reef conservation strategies: An example on north-western Pacific Acropora digitifera," Evol. Appl., no. February, pp. 1-16, 2020.

[89] F. Marin, P. Corstjens, B. De Gaulejac, E. De Vrind-De Jong, and P. Westbroek, "Mucins and molluscan calcification: Molecular characterization of mucoperlin, a novel mucin-like protein from the nacreous shell layer of the fan mussel Pinna nobilis (Bivalvia,

[90] M. Suzuki et al., "An acidic matrix protein, Pif, is a key macromolecule for nacre formation," Science (80-. )., vol. 325, no. 5946, pp. 1388-1390, 2009.

[91] C. Joubert et al., "Transcriptome and proteome analysis of Pinctada margaritifera calcifying mantle and shell: Focus on biomineralization," BMC Genomics, vol. 11, no. 1, 2010.

[92] J. M. Hawkridge, R. K. Pipe, and B. E. Brown, "Localisation of antioxidant enzymes in the cnidarians Anemonia viridis and Goniopora stokesi," Mar. Biol., vol. 137, no. 1, pp. $1-9,2000$. exserta," J. Invertebr. Pathol., vol. 76, no. 3, pp. 176-184, 2000. candidate stress-response genes in the estuarine anemone Nematostella vectensis," Biol. Bull., vol. 214, no. 3, pp. 233-254, 2008. 
826 [95] A. Riesgo et al., "Comparative description of ten transcriptomes of newly sequenced invertebrates and efficiency estimation of genomic sampling in non-model taxa," Front. Zool., vol. 9, pp. 1-24, 2012.

[96] I. Cooke et al., "Signatures of selection in the coral holobiont reveal complex adaptations to inshore environments driven by Holocene climate change," bioRxiv, no. November, 2020.

[97] A. Hoffman and M. Blows, "Species borders: ecological and evolutionary perspectives," TREE, vol. 13, no. 4, pp. 1847-1878, 1994.

[98] T. H. Jorgensen et al., "Linkage disequilibrium and demographic history of the isolated population of the Faroe Islands," Eur. J. Hum. Genet., vol. 10, no. 6, pp. 381-387, 2002.

[99] S. C. H. Barrett, "Understanding plant reproductive diversity," Philos. Trans. R. Soc. B Biol. Sci., vol. 365, no. 1537, pp. 99-109, 2010. populations: from genes to genomes," Curr. Opin. Genet. Dev., vol. 41, pp. 53-61, 2016. local adaptation," New Phytol., 2020. accumulation of deleterious mutations preclude adaptation? Empirical investigation using

845 [103] S. Klopfstein, M. Currat, and L. Excoffier, "The fate of mutations surfing on the wave of a range expansion," Mol. Biol. Evol., vol. 23, no. 3, pp. 482-490, 2006. 
847 [104] T. M. Beissinger, L. Wang, K. Crosby, A. Durvasula, M. B. Hufford, and J. Ross-Ibarra,

848 "Recent demography drives changes in linked selection across the maize genome," Nat.

$849 \quad$ Plants, vol. 2, no. 7, pp. 1-7, 2016.

850 [105] C. Grossen, F. Guillaume, L. F. Keller, and D. Croll, "Purging of highly deleterious mutations through severe bottlenecks in Alpine ibex," Nat. Commun., vol. 11, no. 1, 2020.

[106] A. Tellier et al., "Fitness effects of derived deleterious mutations in four closely related wild tomato species with spatial structure," Heredity (Edinb)., vol. 107, no. 3, pp. 189199, 2011.

[107] M. C. Lecheta et al., "Integrating GWAS and Transcriptomics to Identify the Molecular Underpinnings of Thermal Stress Responses in Drosophila melanogaster," Front. Genet., vol. 11, no. June, pp. 1-17, 2020.

[108] D. Berger, J. Stångberg, J. Baur, and R. J. Walters, “Elevated temperature increases genome-wide selection on de novo mutations," Proc. R. Soc. B Biol. Sci., vol. 288, no.

[109] K. Maor-Landaw et al., "Mediterranean versus Red sea corals facing climate change, a transcriptome analysis," Sci. Rep., vol. 7, p. 42405, 2017. 


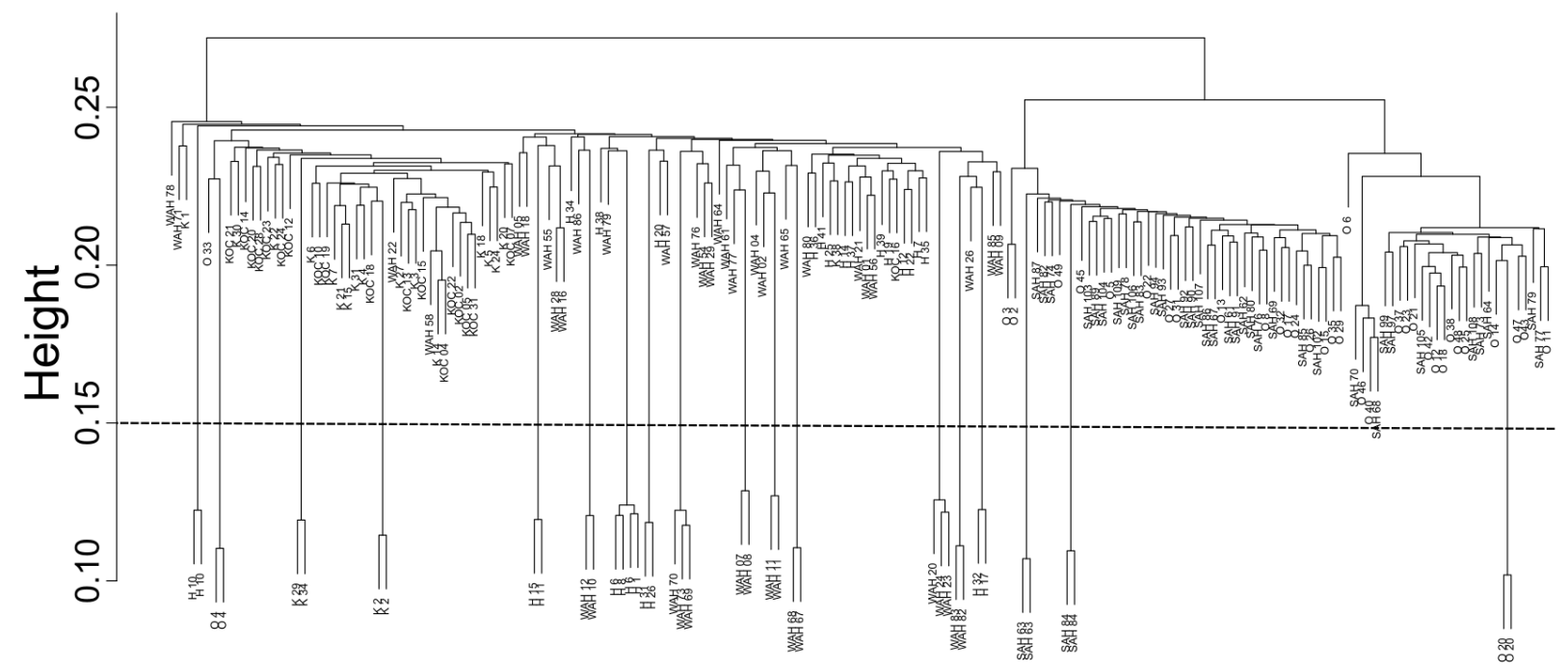

Supplemental Figure 1. Identity by state dendrogram, dotted line represents cutoff for clone assignment.

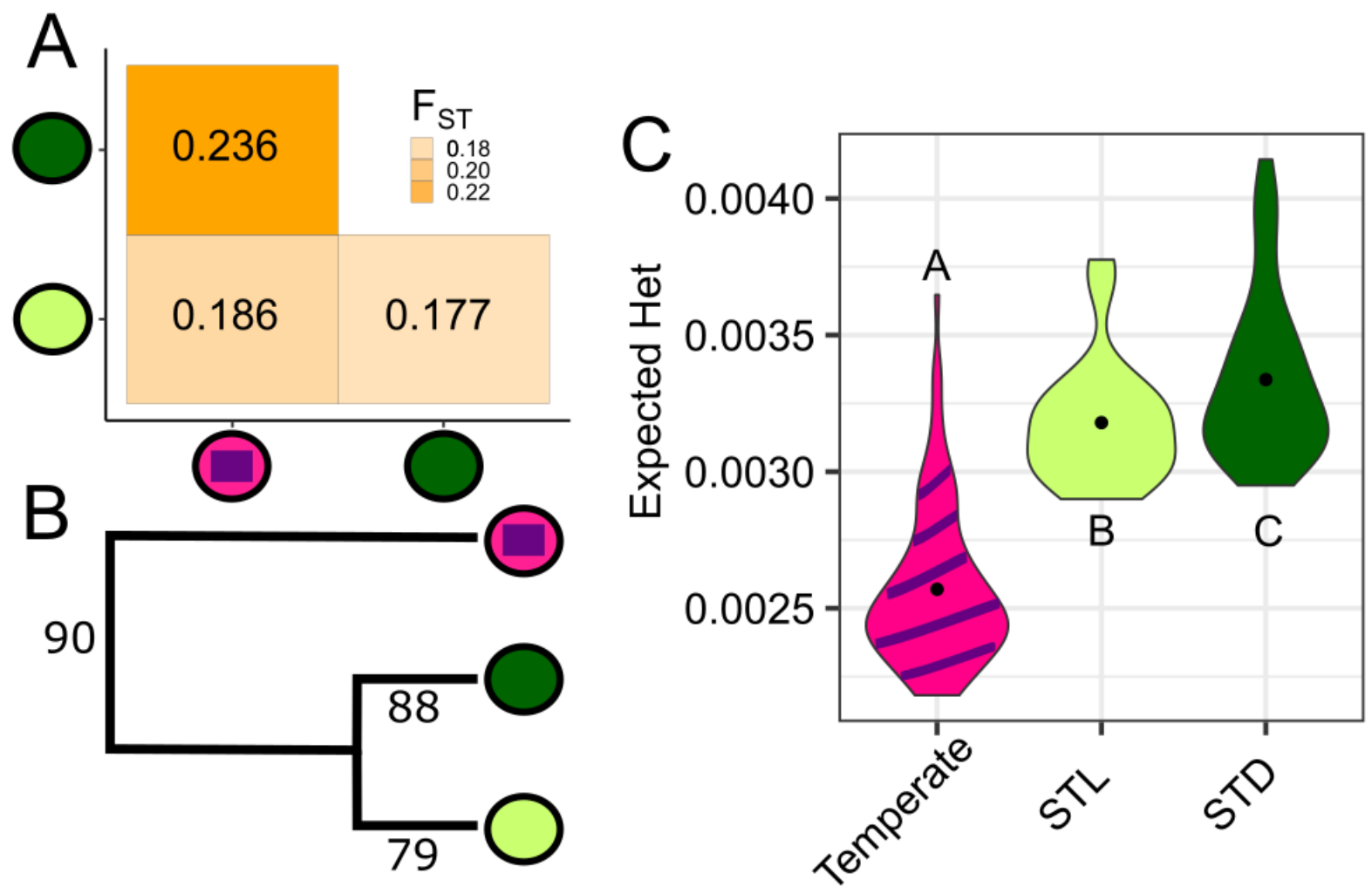

Supplemental Figure 2. A) Pairwise global $\mathrm{F}_{\mathrm{ST}}$ between all three lineages. B) RAXML tree. Numbers correspond to branch bootstrap support values. C) Expected heterozygosity estimates for each Acropora hyacinthus lineage. Different letters denote significant differences $(\mathrm{p}<0.05)$ between lineages. 


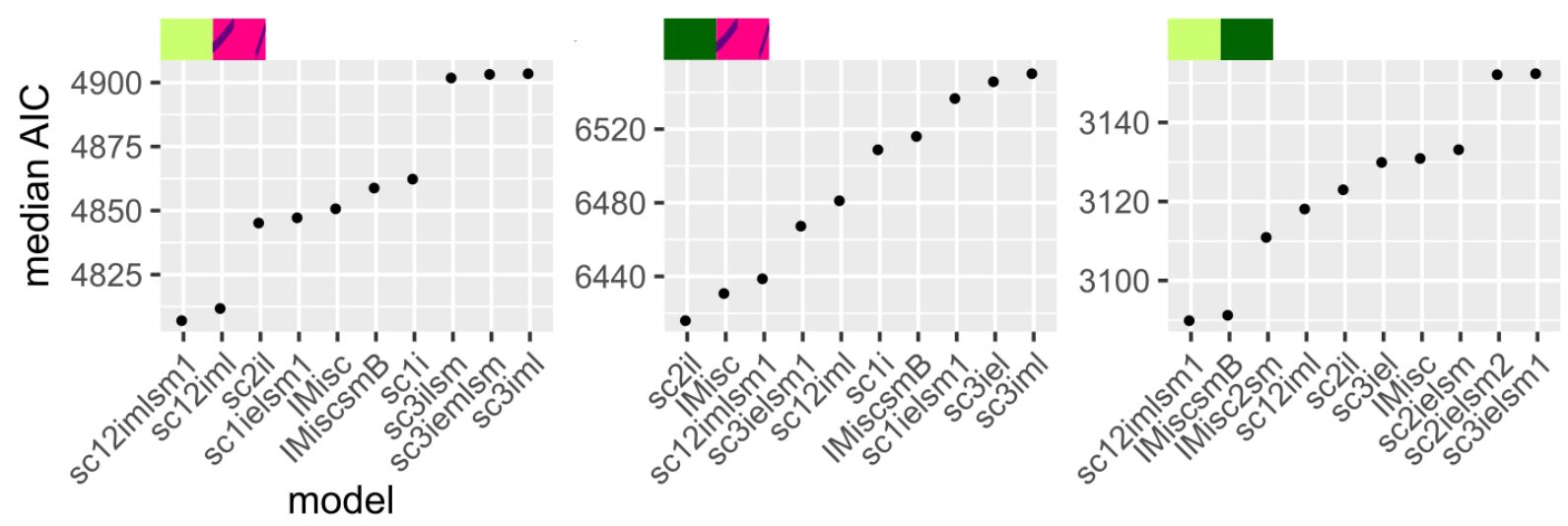

Supplemental Figure 3. Median AIC scores for top 10 models predicted by moments. Model descriptions in Supplementary Table 2. For a complete list of all models conducted please see:

https://github.com/z0on/AFS-analysis-withmoments/blob/master/multimodel inference/moments multimodels.xlsx
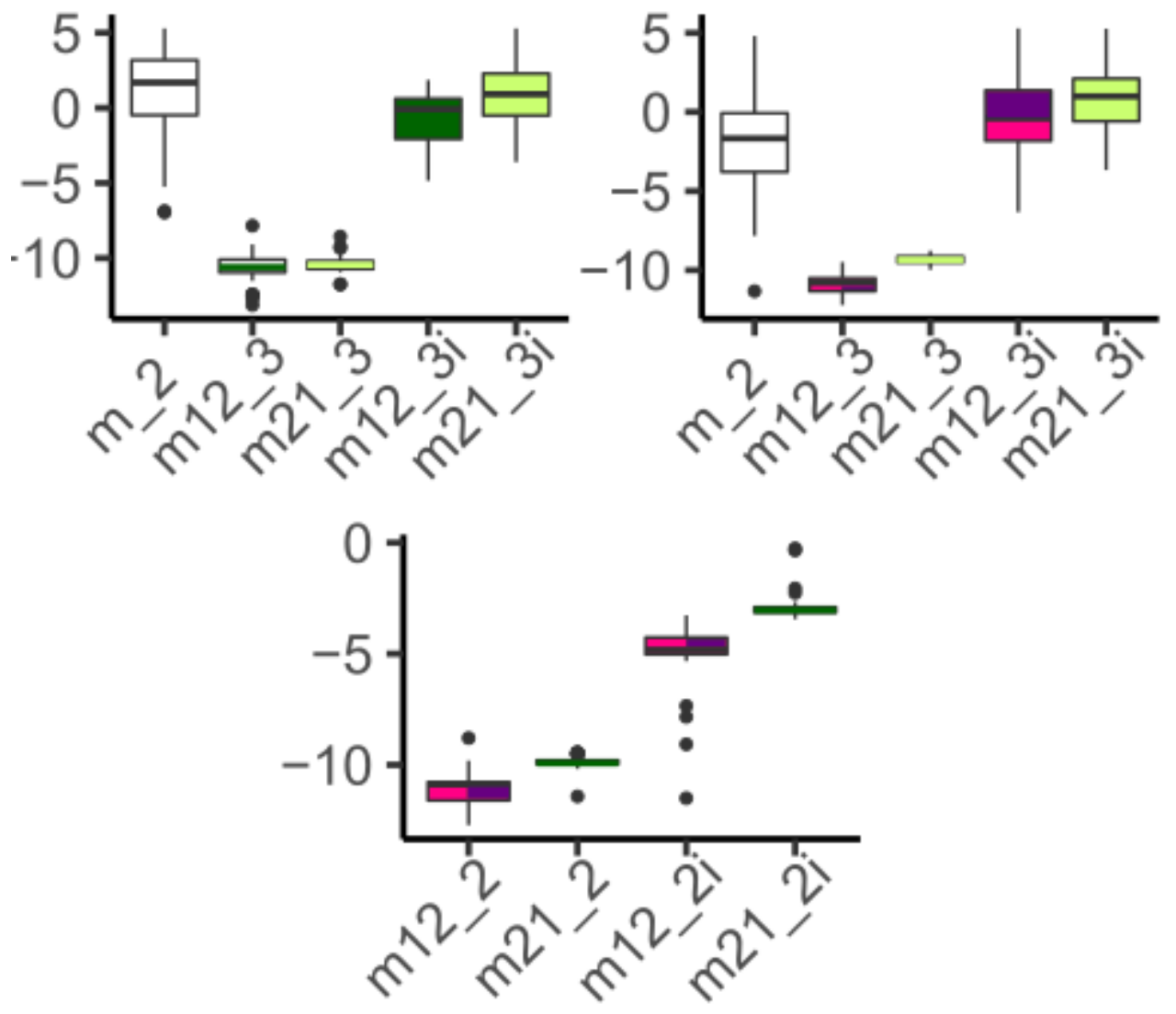
Supplemental Figure 4. Log transformed migration rates from pairwise moments parameter output for Acropora hyacinthus lineage comparisons. Boxes are colored by migrant origin; i represents migration rates for genomic islands; _2 is 2nd epoch;_3 3rd epoch.
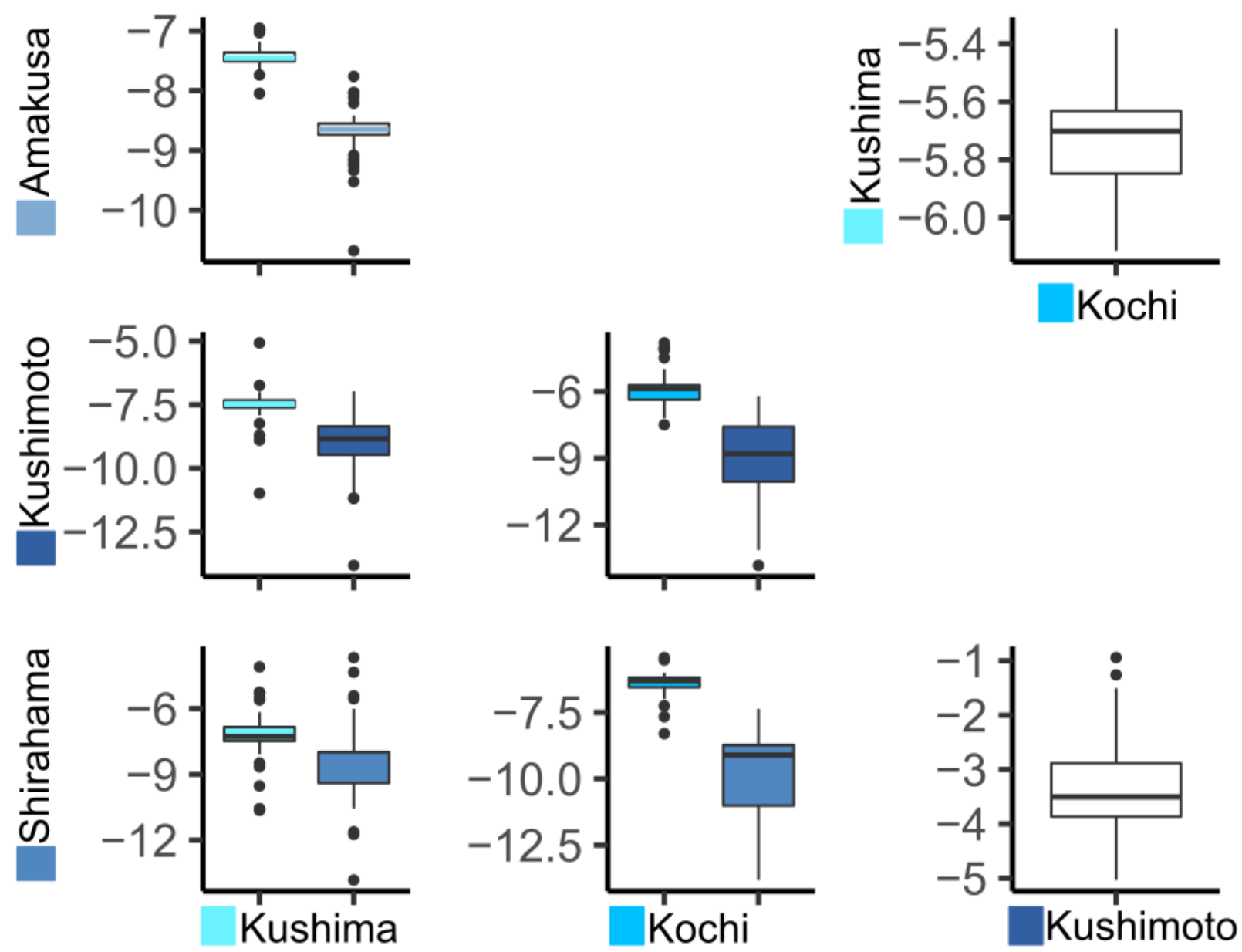

Supplemental Figure 5. Mean migration rates for all pairwise sites within mainland Japan for the temperate Acropora hyacinthus lineage. Color corresponds to the source of migrants. Log transformed migration rates from pairwise moments parameter output for lineage comparisons. Boxes are colored by migrant origin; plots with single migration parameters represent symmetrical migration rates. 

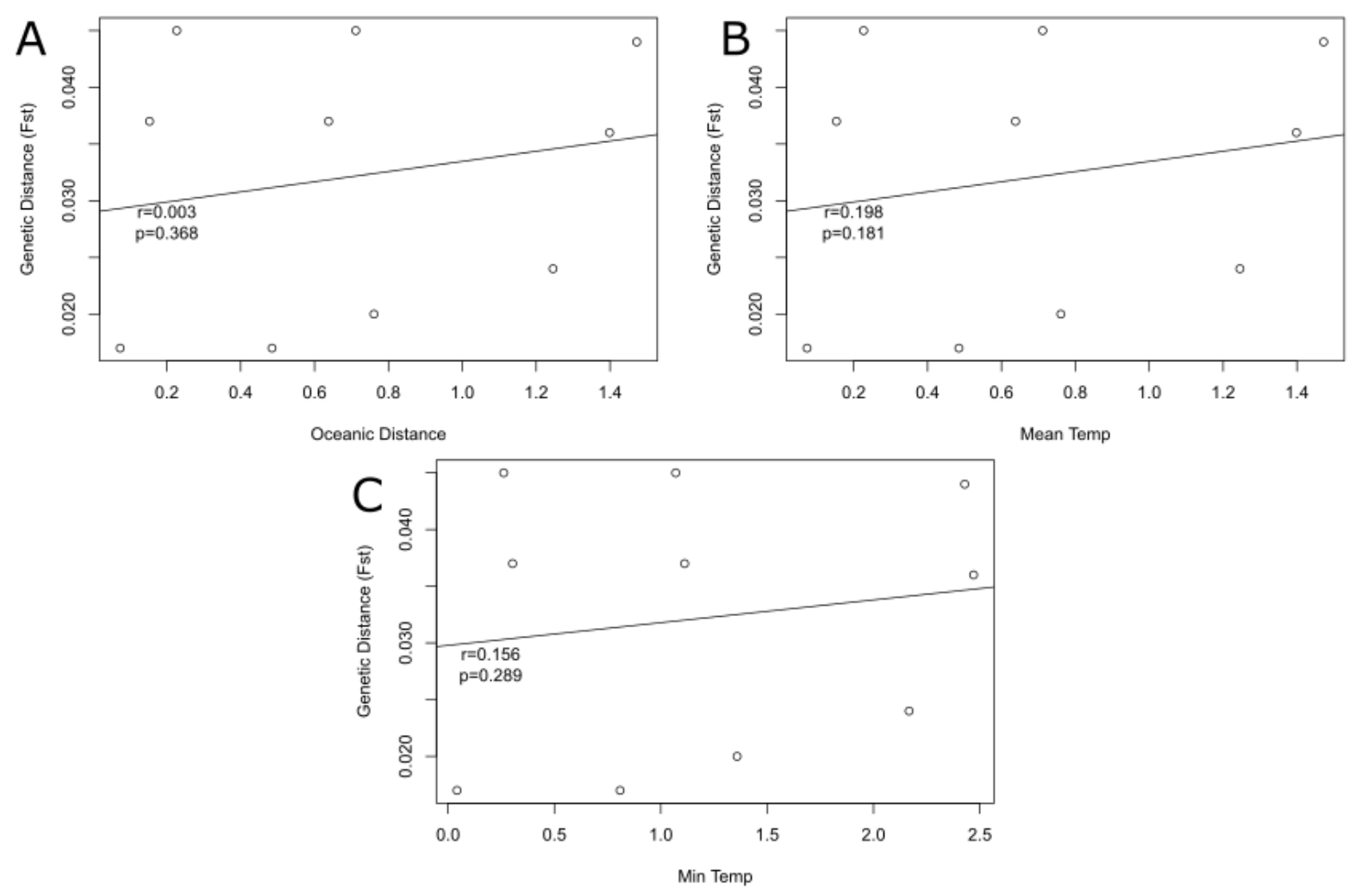

Supplemental Figure 6. Relationships between pairwise genetic distances and A) Oceanic distances (Nakabayashi et al 2019), B) Mean monthly sea surface temperature (SST), and C) Minimum monthly SST. 


\begin{tabular}{|c|c|c|c|c|c|c|c|c|c|c|}
\hline Site & site_lat & site_long & $\begin{array}{l}\text { Depth } \\
\text { (m) }\end{array}$ & \#sampled & \#sequenced & $\begin{array}{l}\text { \#w/o } \\
\text { clones }\end{array}$ & $\begin{array}{l}\text { Date } \\
\text { sampled }\end{array}$ & $\begin{array}{l}\text { \#Temperate } \\
\text { Lineage }\end{array}$ & \#STD & \#STL \\
\hline Kushima & 31.46454 & 131.2283 & NA & 38 & 21 & 20 & Dec-14 & 20 & 0 & 0 \\
\hline $\begin{array}{l}\text { Sekesei } \\
\text { Lagoon }\end{array}$ & 24.32921 & 124.0444 & $1.0-6.6$ & 51 & 40 & 37 & Jul-14 & 0 & 27 & 7 \\
\hline Amakusa & 32.4585 & 130.1934 & NA & 42 & 26 & 18 & Sep-09 & 18 & 0 & 0 \\
\hline Kushimoto & 33.47247 & 135.7815 & $2.4-5.5$ & 31 & 23 & 17 & Jun-16 & 17 & 0 & 0 \\
\hline Shirahama & 33.511 & 135.3482 & $0.7-9.4$ & 32 & 21 & 19 & Jun-16 & 19 & 0 & 0 \\
\hline Oura Bay & 26.53717 & 128.0579 & NA & 50 & 38 & 36 & Mar-13 & 2 & 14 & 16 \\
\hline Kochi & 32.78871 & 132.8705 & $1.0-6.0$ & 33 & 19 & 19 & Oct-11 & 19 & 0 & 0 \\
\hline Total & & & & 277 & 188 & 166 & & 95 & 41 & 23 \\
\hline
\end{tabular}

Supplemental Table 1. Sample information for all collections.

\begin{tabular}{|c|c|c|c|c|c|}
\hline Name & Split & Epochs & Genomic islands & Migration epochs & Symmetric migration? \\
\hline sc12imlsm & 1 & $\begin{array}{l}\text { 1before split, } \\
\text { then } 2\end{array}$ & 1 & 2,3 & yes \\
\hline sc12iml & 1 & $\begin{array}{l}\text { 1before split, } \\
\text { then } 2\end{array}$ & 1 & 2,3 & no \\
\hline sc2il & 1 & 2 & 1 & 2 & no \\
\hline sc1ielsm1 & 1 & 1 & 1 & 1 & $\begin{array}{l}\text { yes in } 1 \text { st epoch, no in } 3 d \\
\text { epoch }\end{array}$ \\
\hline IMisc & 1 & 2 & 1 & 1 & no \\
\hline IMiscsmB & 1 & 2 & 1 & 1 & $\begin{array}{l}\text { yes for background genome, } \\
\text { no for islands }\end{array}$ \\
\hline sc1i & 1 & 1 & 1 & $\begin{array}{l}1 \text { early and late, } \\
\text { independent }\end{array}$ & 0 \\
\hline sc3ilsm & 1 & 3 & 1 & 3 & yes \\
\hline sc3iemlsm & 1 & 3 & 1 & $1,2,3$ & yes \\
\hline sc3iml & 1 & 3 & 1 & 2,3 & 0 \\
\hline sc3iel & 1 & 3 & 1 & 1,3 & 0 \\
\hline IMisc2sm & 1 & $\begin{array}{l}2, \text { free size of } \\
1 \text { st epoch }\end{array}$ & 1 & 1 & yes \\
\hline sc2ielsm & 1 & 2 & 1 & 1,2 & yes \\
\hline sc2ielsm2 & 1 & 2 & 1 & 1,2 & $\begin{array}{l}\text { no in 1st epoch, yes in 2nd } \\
\text { epoch }\end{array}$ \\
\hline
\end{tabular}

Supplementary Table 2. Model descriptions for models with top 10 median AICs in Supplementary Figure 3. 
SupplementabiERReiv1preprint doi: https://doi.org/10.1101/2021.06.17.448885; this version posted June 17, 2021. The copyright holder for this preprint (which was not certified by peer review) is the author/funder, who has granted bioRxiv a license to display the preprint in perpetuity. It is made available under aCC-BY-NC-ND 4.0 International license.

Click here to access/download Supplemental Videos and Spreadsheets Japan Rad Supplemental File 1.xlsx 